\title{
Linear stability of the flow of a second order fluid past a wedge
}

Cite as: Phys. Fluids 32, 084102 (2020); https://doi.org/10.1063/5.0018300

Submitted: 13 June 2020 . Accepted: 13 July 2020 . Published Online: 03 August 2020

M. Cracco (D), C. Davies (D), and T. N. Phillips (D)

\section{ARTICLES YOU MAY BE INTERESTED IN}

Effects of partial slip on the local-global linear stability of the infinite rotating disk boundary layer

Physics of Fluids 32, 074105 (2020); https://doi.org/10.1063/5.0012976

The dispersion of spherical droplets in source-sink flows and their relevance to the

COVID-19 pandemic

Physics of Fluids 32, 083302 (2020); https://doi.org/10.1063/5.0021427

Flow structures in transitional and turbulent boundary layers

Physics of Fluids 31, 111301 (2019); https://doi.org/10.1063/1.5121810

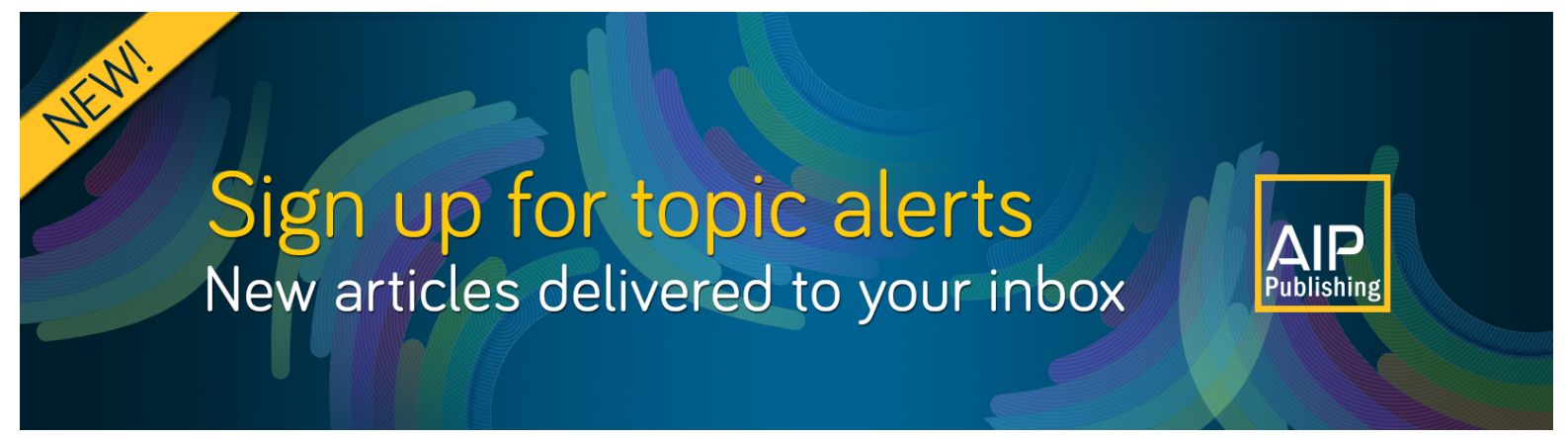




\title{
Linear stability of the flow of a second order fluid past a wedge
}

\author{
Cite as: Phys. Fluids 32, 084102 (2020); doi: 10.1063/5.0018300 \\ Submitted: 13 June 2020 - Accepted: 13 July 2020 • \\ Published Online: 3 August 2020
}

M. Cracco, a) (D) C. Davies, (D) and T. N. Phillips (D)

AFFILIATIONS
School of Mathematics, Cardiff University, Cardiff CF24 4AG, United Kingdom

a) Author to whom correspondence should be addressed: martina.cracco@yahoo.it

\begin{abstract}
The linear stability analysis of Rivlin-Ericksen fluids of second order is investigated for boundary layer flows, where a semi-infinite wedge is placed symmetrically with respect to the flow direction. Second order fluids belong to a larger family of fluids called order fluids, which is one of the first classes proposed to model departures from Newtonian behavior. Second order fluids can model non-zero normal stress differences, which is an essential feature of viscoelastic fluids. The linear stability properties are studied for both signs of the elasticity number $K$, which characterizes the non-Newtonian response of the fluid. Stabilization is observed for the temporal and spatial evolution of two-dimensional disturbances when $K>0$ in terms of increase of critical Reynolds numbers and reduction of growth rates, whereas the flow is less stable when $K<0$. By extending the analysis to three-dimensional disturbances, we show that a positive elasticity number $K$ destabilizes streamwise independent waves, while the opposite happens for $K<0$. We show that, as for Newtonian fluids, the non-modal amplification of streamwise independent disturbances is the most dangerous mechanism for transient energy growth, which is enhanced when $K>0$ and diminished when $K<0$.

(C) 2020 Author(s). All article content, except where otherwise noted, is licensed under a Creative Commons Attribution (CC BY) license (http://creativecommons.org/licenses/by/4.0/). https://doi.org/10.1063/5.0018300
\end{abstract}

\section{INTRODUCTION}

The mechanical behavior of many real fluids is described by Navier-Stokes theory. This theory is based on the assumption of a Newtonian constitutive equation. More specifically, the extra-stress tensor is expressed as a linear, isotropic function of the components of the velocity gradient. Many common fluids, such as water and air, can be assumed to be Newtonian. However, many rheologically complex fluids, such as polymer solutions, soaps, blood, paints, shampoo, and ketchup, exhibit a variety of phenomena that cannot be adequately described by a Newtonian constitutive equation.

Viscoelastic fluids are examples of non-Newtonian fluids, and they exhibit both viscous and elastic properties when undergoing deformation. Elasticity is the tendency of the material to return to its original shape once the external force is removed. Viscoelastic fluids undergo a gradual deformation and recovery when they are subjected to loading and unloading. The stress is directly proportional to neither the strain nor the rate of strain, and the relationship is more complex.
In this paper, we consider a subclass of differential type fluids known as the Rivlin-Ericksen fluids of second order. ${ }^{1}$ In these models, only an infinitesimal part of the history of the deformation gradient has an influence on the stress. The extra stress is a function of the velocity gradient and its higher time derivatives. These materials lack a gradually fading memory, and they cannot represent the phenomenon of stress relaxation. However, they can predict non-zero normal stress differences, which is an important feature of viscoelastic fluids. As models to describe viscoelastic fluids, order fluids are suitable to describe slightly elastic fluids, where the fluid is only a small departure from the Newtonian fluid and flows for which the Rivlin-Ericksen tensors vary slowly. ${ }^{2}$

In theoretical work, Rajagopal et al. ${ }^{3}$ showed that it is possible to apply Prandtl's boundary layer theory to the case of a nonNewtonian fluid of second order. In particular, they showed that the equations of motion of a second order fluid can be satisfied by an irrotational flow, and they identified suitable assumptions to obtain a consistent theory. In the case of fluids of a differential type, the equations of motion are an order higher than the Navier-Stokes 
equations, and thus, the no-slip and no-penetration boundary conditions are insufficient to determine the solution completely. ${ }^{4,5}$ The same is also true for the boundary layer approximation. In order to overcome this difficulty, in their study of an incompressible fluid of liquid $\mathrm{B}^{\prime}$ near a stagnation point, Beard and Walters ${ }^{6}$ suggested a perturbation method. This method was also adopted by Rajagopal et al. ${ }^{7}$ in their analysis of the flow past a wedge of an incompressible fluid of second grade. The perturbation method reduces the order of the problem but is only valid for small values of the nonNewtonian parameter. This parameter multiplies the higher order spatial derivatives in the equation.

While studying flow near a stagnation point and flow past a wedge, Garg and Rajagopal ${ }^{8,9}$ suggested that it would be preferable to use an augmented boundary condition justified by physically reasonable assumptions, also adopted by Vajravelu and Roper, ${ }^{10}$ and by Vajravelu and Rollins. ${ }^{11}$

Another difficulty that arises is the impossibility of finding a similarity solution to the boundary layer equations as in the Newtonian case, with the exception of stagnation flow. In this paper, we apply a pseudo-similarity transformation ${ }^{9}$ to obtain the mean flow, which will be the starting point of the linear stability analysis.

The aim of this paper is to understand the stability properties of second order fluids in boundary layers. Specifically, a configuration of flow over a semi-infinite wedge is investigated (Fig. 1). One important motivation for studying the stability behavior of viscoelastic fluids, and in particular, polymer suspensions can be found in drag reduction in turbulent regime. ${ }^{12-14}$ This phenomenon was first observed over 70 years ago. In turbulent boundary layers, dissolving a small quantity of long-chain flexible polymers in solution can reduce turbulent friction by a significant amount. For a summary of the historical findings and recent developments, we refer to the review by $\mathrm{Xi}$.

First, we perform a classical linear stability analysis by solving the linearized system of equations around the steady mean flow, which is assumed to be parallel. The mean flow and the stability equations are solved numerically. For two-dimensional disturbances, the results are represented in terms of temporal growth rates, neutral stability curves, and critical Reynolds numbers. Later, the linear stability analysis is extended to three-dimensional disturbances.

Classical linear stability analysis is based on eigenvalues. However, in hydrodynamic stability and many other physical situations dominated by non-normal systems, eigenvalues prove to be

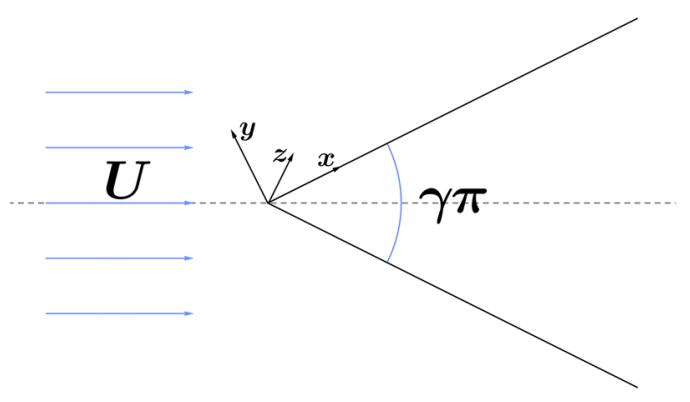

FIG. 1. Semi-infinite wedge flow configuration $(\gamma>0)$. misleading, and they do not describe correctly the whole dynamics. ${ }^{16}$ In non-normal systems, such as Poiseuille, Couette, and Blasius flows, there can be short-time growth of energy even if all the eigenvalues decay exponentially. ${ }^{17}$ This phenomenon is known as transient growth.

For Newtonian fluids, the possibility of transient growth has been known since the 1980s. ${ }^{18}$ Some previous work has investigated transient growth of viscoelastic fluids in channel flows. ${ }^{19}$ In this paper, we extend the analysis and consider the transient growth of second order fluids in boundary layers.

\section{SECOND ORDER FLUIDS} form

The Cauchy stress tensor $\boldsymbol{\sigma}$ in a fluid of second order has the

$$
\boldsymbol{\sigma}=-p \mathbf{I}+\mu \mathbf{A}_{1}+\alpha_{1} \mathbf{A}_{2}+\alpha_{2} \mathbf{A}_{1}^{2},
$$

where $p$ is the pressure, $\mu$ is the dynamic viscosity, and $\alpha_{1}$ and $\alpha_{2}$ (SI: $\mathrm{kg} / \mathrm{m}$ ) are the material moduli usually referred to as normal stress moduli. The spherical stress $-p \mathbf{I}$ is due to the constraint of incompressibility, while $\mathbf{A}_{1}$ and $\mathbf{A}_{2}$ are the Rivlin-Ericksen tensors of order 1 and order 2 , respectively, defined by

$$
\mathbf{A}_{1}=\nabla \mathbf{v}+\nabla \mathbf{v}^{T}, \quad \mathbf{A}_{2}=\frac{\mathrm{DA} \mathbf{A}_{1}}{\mathrm{D} t}+(\nabla \mathbf{v}) \mathbf{A}_{1}+\mathbf{A}_{1}(\nabla \mathbf{v})^{T},
$$

where $\mathbf{v}$ denotes the velocity field and $\mathrm{D} / \mathrm{D} t$ denotes the material time derivative.

The sign of the material parameters in this model has been a source of some controversy. ${ }^{20}$ In this paper, we consider both cases $\alpha_{1}>0$ and $\alpha_{1}<0$. The second order model with $\alpha_{1}>0$ is studied because of its compatibility with thermodynamics. Since the form (1) is frame-indifferent, it can be used as an exact model. In this view, Dunn and Fosdick ${ }^{21}$ and Fosdick and Rajagopal ${ }^{22}$ justified some assumptions on the coefficients of the second order constitutive equation. In order for the fluid model to be compatible with thermodynamics, in the sense that all motions of the fluid satisfy the Clausius-Duhem inequality and the assumption that the specific Helmholtz free energy be a minimum in equilibrium, it is necessary that

$$
\mu \geq 0, \quad \alpha_{1} \geq 0, \quad \text { and } \quad \alpha_{1}+\alpha_{2}=0 .
$$

A detailed discussion of these assumptions can be found in the critical review of Dunn and Rajagopal.

The second order model with $\alpha_{1}<0$ is studied because it gives the right sign for the first normal stress difference. ${ }^{2}$ Moreover, in terms of linear stability, it is a consistent approximation to a stressrelaxing fluid, such as the Maxwell fluid, at small elasticity numbers and when the disturbance time scale is large compared to the characteristic time scale of the fluid. ${ }^{23}$

To the best of our knowledge, little work has been performed on the stability of second order fluids in boundary layers unlike the situation for channel flows. Chun and Schwarz ${ }^{24}$ studied the stability of the plane Poiseuille flow of a second order fluid $\left(\alpha_{1}\right.$ $<0)$. Their analysis yields an Orr-Sommerfeld equation modified by adding a non-Newtonian term. They showed that the critical Reynolds number decreases as the magnitude of the non-Newtonian 
parameter increases. Later, Sadeghy et al. ${ }^{25}$ solved the same modified Orr-Sommerfeld equation for the plane Poiseuille flow of a second order fluid $\left(\alpha_{1}>0\right)$. They showed that non-Newtonian effects in this model are stabilizing.

Regarding the stability of other viscoelastic fluids, some results were obtained for channel flows but, to the best of our knowledge, not much has been done for boundary layer flows. Porteous and Denn $^{23}$ studied the linear stability analysis of plane Poiseuille flow for the second order $\left(\alpha_{1}<0\right)$ and Maxwell fluids. They showed a destabilization process due to elasticity. At high values of the elasticity number, the stability is qualitatively different from that for Newtonian fluids because it results from the second mode of the Orr-Sommerfeld equation.

Sureshkumar and Beris ${ }^{26}$ used an Arnoldi-based orthogonalization algorithm to investigate the linear stability of Poiseuille flow. The models investigated are Upper Convected Maxwell (UCM), Oldroyd-B, and Chilcott-Rallison fluids. The results show that the destabilization caused by elasticity for the UCM fluid is reduced when the effects of solvent viscosity and finite extensibility are taken into account. Zhang et al. ${ }^{27}$ showed that when the polymer relaxation time is shorter than the instability time scale, the Poiseuille flow of FENE-P fluids appears to be less stable. However, in the opposite case, the strong elastic effect stabilizes the flow.

\section{GOVERNING EQUATIONS}

The field equations for an incompressible second order fluid can be derived by substituting expression (1) for the Cauchy stress into the balance of linear momentum,

$$
\rho \frac{\mathrm{D} \mathbf{v}}{\mathrm{D} t}=\nabla \cdot \boldsymbol{\sigma}
$$

where $\rho$ is the density of the fluid. Since the fluid is incompressible, we require all possible motions be isochoric, and hence, the continuity equation reduces to

$$
\nabla \cdot \mathbf{v}=0
$$

The geometric configuration considered consists of a wedge of angle $\gamma \pi$, which is placed symmetrically with respect to the direction of the uniform velocity field, as shown in Fig. 1 for $y>0$. The $x$-axis is chosen to be in the streamwise direction, the $z$-axis in the spanwise direction, and the $y$-axis in the wall-normal direction. Due to the symmetric nature of the problem, we can restrict our analysis to the case $y \geq 0$. Note that if $\gamma=0$, we recover the case of flow over a semiinfinite flat plate, while $\gamma=1$ corresponds to the case of a stagnation point flow. When $\gamma>1$, we have the flow into an acute corner, $\gamma<0$ gives a flow past a corner, and $0<\gamma<1$ is the flow past an acute wedge.

\section{MEAN FLOW}

Applying the boundary layer approximation to the field equations (4) and (5) in the same way it is done for Newtonian fluids, we obtain

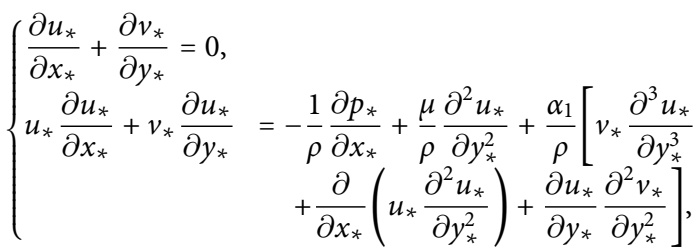

where starred dependent and independent variables indicate dimensional variables. If the plate forms an angle $\gamma \pi / 2$ with respect to the uniform velocity field (Fig. 1), the free-stream velocity varies with distance to the leading edge according to potential flow theory ${ }^{28}$ as a power law,

$$
U_{e}\left(x_{*}\right)=a x_{*}^{m},
$$

where $a$ is a positive constant and the exponent $m$ is related to the angle parameter: $\gamma=2 m /(m+1)$.

After the boundary layer transformation,

$$
\eta=\frac{y_{*}}{\delta}, \quad \psi_{*}=\delta U_{e}\left(x_{*}\right) f
$$

where

$$
\delta=\sqrt{\frac{v}{a(m+1)} x_{*}^{\frac{1-m}{2}}}
$$

is a measure for the displacement thickness and $\psi_{*}$ is the stream function, the boundary layer equations (6) are transformed into the following local ordinary differential equation (ODE):

$$
\begin{aligned}
2(m+1) f^{\prime \prime \prime} & +(m+1) f f^{\prime \prime}+2 m-2 m\left(f^{\prime}\right)^{2} \\
= & \frac{\alpha_{1} a}{\rho v}(m+1) x_{*}^{m-1}\left[(m+1) f^{i v} f\right. \\
& \left.+2(1-3 m) f^{\prime} f^{\prime \prime \prime}+(3 m-1)\left(f^{\prime \prime}\right)^{2}\right],
\end{aligned}
$$

where' indicates the derivative with respect to the boundary layer variable, $\eta$. The key idea is to solve Eq. (9) numerically for fixed values of $x_{*}$ in order to obtain a local solution. It can be easily seen that a similarity solution is possible only for stagnation point flow, ${ }^{8,9}$ where $m=1$. For the stability analysis, Eq. (9) will be transformed and the dependency on the streamwise position $x_{*}$ will be included in the elasticity parameter, which will be defined later in this section.

The stability analysis is traditionally performed, for a Newtonian fluid, by choosing a fixed streamwise position $x_{*}=x_{0}$, as first proposed by Tollmien. ${ }^{29}$ The approach consists of finding the longitudinal velocity at that station, ignoring the relatively small transverse velocity, and then solving the Orr-Sommerfeld equation for the resulting base profile.

Following the example of Schmid and Henningson, ${ }^{30}$ we apply the same procedure to the second order fluid and we define a displacement thickness, $\delta_{0}$, at position $x_{0}$, as follows:

$$
\delta_{0}=C \sqrt{\frac{v}{a(m+1)}} x_{0}^{\frac{1-m}{2}},
$$

where $C$ is a constant given by

$$
C=\int_{0}^{\infty}\left(1-f_{\text {Newt }}^{\prime}(\eta)\right) d \eta
$$


calculated in the Newtonian case. This choice was made in order to easily compare non-Newtonian solutions with Newtonian solutions. The Reynolds number $R e_{0}=U_{e}\left(x_{0}\right) \delta_{0} / v$ based on the displacement thickness satisfies the following relation:

$$
\frac{x_{0}}{\delta_{0}}=\frac{m+1}{C^{2}} R e_{0}
$$

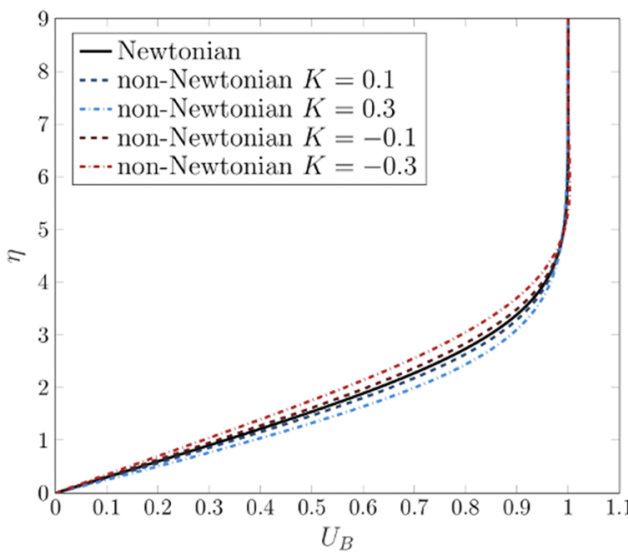

(a)

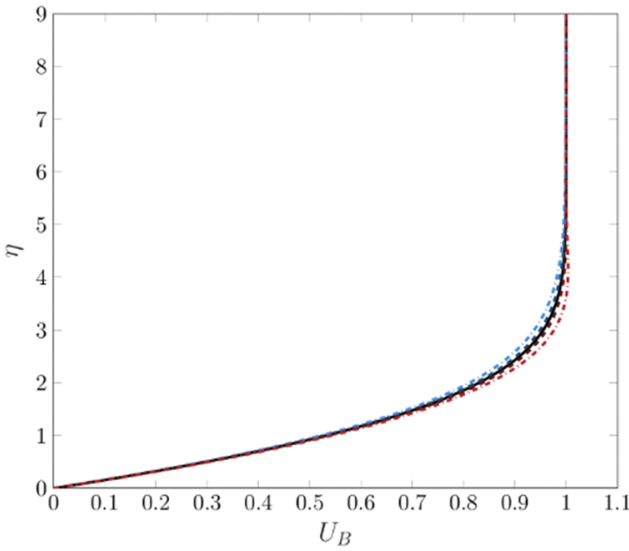

(c)

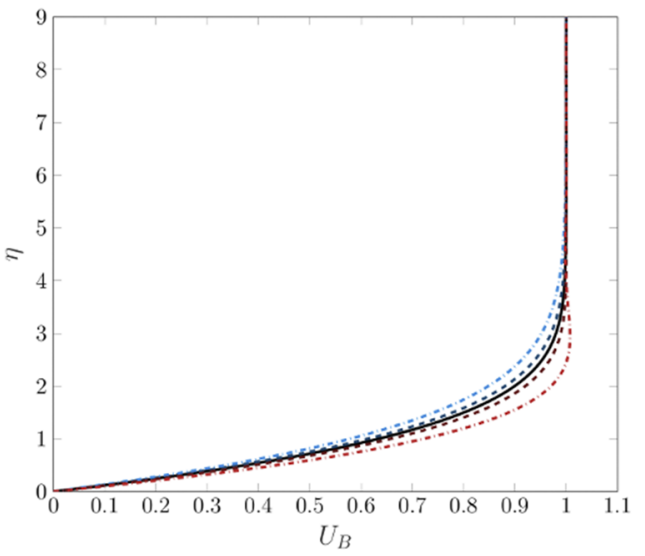

(e)

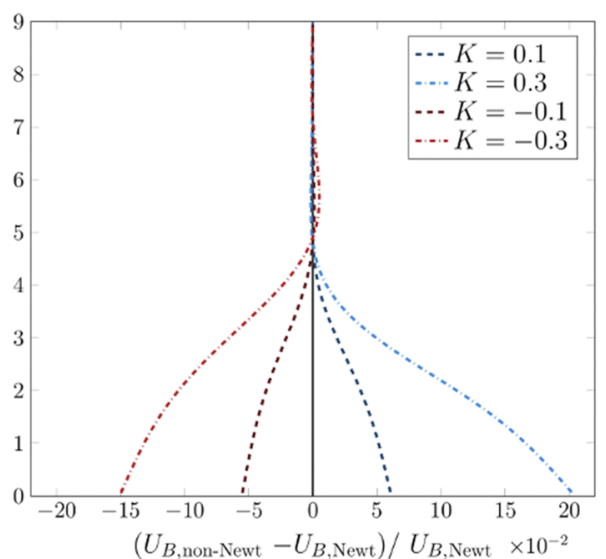

(b)

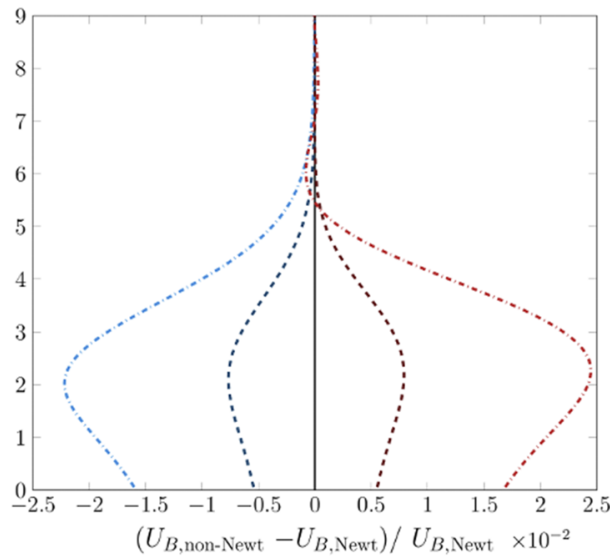

(d)

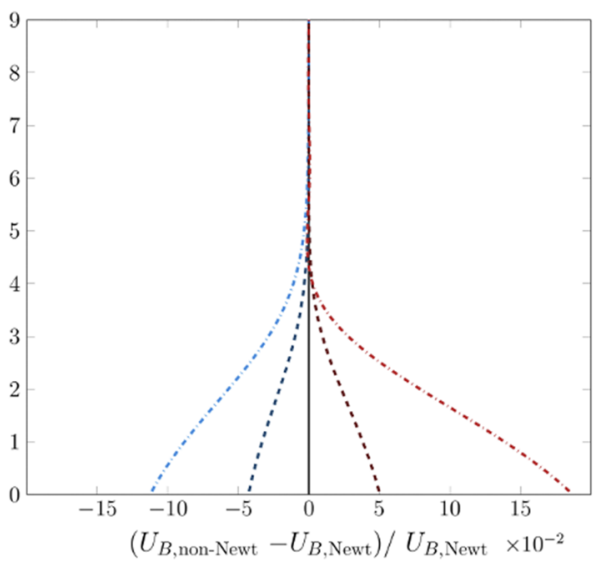

(f)
Using relation (12), Eq. (9) at the fixed position $x_{0}$ can be rewritten as

$$
\begin{aligned}
2(m+1) f^{\prime \prime \prime} & +(m+1) f f^{\prime \prime}+2 m-2 m\left(f^{\prime}\right)^{2} \\
& =K_{0} C^{2}\left[(m+1) f^{i v} f+2(1-3 m) f^{\prime} f^{\prime \prime \prime}+(3 m-1)\left(f^{\prime \prime}\right)^{2}\right],
\end{aligned}
$$

FIG. 2. Velocity profile and relative variation with respect to the Newtonian profile for increasing and decreasing values of the parameter $K=K_{0} C^{2}$. [(a) and (b)] $\gamma$ $=0$ (flat plate), [(c) and (d)] $\gamma=0.5$ (flow past a wedge), and [(e) and (f)] $\gamma=1$ (stagnation flow) 
where $K_{0}=\alpha_{1} /\left(\rho \delta_{0}^{2}\right)$ is a non-dimensional parameter known as the elasticity number that can be interpreted as representing the ratio of non-Newtonian normal stress forces to inertial forces. In fact, $K_{0}=W i_{0} / R e_{0}$, where $W i_{0}=\alpha_{1} U_{e}\left(x_{0}\right) /\left(\mu \delta_{0}\right)$ is the Weissenberg number.

In order to have a valid boundary layer theory for nonNewtonian fluids of second order, it is necessary that not only the ratio of the inertial forces to the forces due to the tangential stresses be large (high Reynolds number), as in the Newtonian case, but also the ratio of the inertial forces to the forces due to the normal stresses should be large. ${ }^{3}$ This implies the following assumptions:

$$
R e_{0} \gg 1 \text { and }\left|K_{0}\right| \ll 1 \text {. }
$$

The mean flow for the stability analysis is non-dimensionalized using the free-stream velocity $U_{e}\left(x_{0}\right)$, defined by $(7)$, at the streamwise location $x_{0}$. Hence, the velocity in the $x$-direction is

$$
U_{B}=\frac{u_{*}}{U_{e}\left(x_{0}\right)}=f^{\prime}
$$

The wall-normal velocity $V_{B}$ is

$$
V_{B}=\frac{v_{*}}{U_{e}\left(x_{*}\right)}=\frac{1}{2} \frac{C}{(m+1) R e_{0}}\left[(1-m) \eta f^{\prime}-(m+1) f\right] .
$$

It is clear that this flow is nearly parallel because the transverse velocity $V_{B}$ is smaller than $U_{B}$ by a factor of $R e_{0}^{-1}$, so it will be neglected in order to perform the stability analysis. This is a valid approximation when the Reynolds number $R e_{0}$ based on the displacement thickness is large.

Equation (13) is solved by applying the usual boundary conditions that ensure no-slip and no-penetration at the wall and matching with the free-stream velocity at infinity,

$$
\begin{aligned}
f(\eta)=0, f^{\prime}(\eta)=0 & \text { at } \eta=0, \\
f^{\prime}(\eta) \rightarrow 1 & \text { as } \eta \rightarrow \infty,
\end{aligned}
$$

augmented by the condition

$$
f^{\prime \prime}(\eta) \rightarrow 0 \quad \text { as } \eta \rightarrow \infty
$$

The additional boundary condition (15) is required since the differential equation (13) is of fourth order. Condition (15) is derived by imposing $\frac{\partial u_{*}}{\partial y_{*}} \rightarrow 0$ at infinity and is equivalent to requiring that the solution approaches the free-stream velocity smoothly far from the wall. $^{8,9}$

The effect of elasticity on the velocity profile changes with the geometrical configuration. For $K_{0}>0$, we can see from Figs. 2(a) and 2 (b) that the velocity at all points in the boundary layer is larger in the non-Newtonian case for the flow over a flat plate $(\gamma=0)$ and the greater variation appears at the wall. Instead, for the second order model with $K_{0}<0$, the velocity at all points in the boundary layer is smaller in the non-Newtonian case for flow over a flat plate. Figures 2(c) and 2(d) show that, for a wedge angle of $\pi / 2$, there is a smaller relative variation than for the flat plate observed in Figs. 2(a) and 2(b). When $K_{0}>0$, the non-Newtonian velocity is slightly smaller inside the boundary layer, while when $K_{0}<0$, the non-Newtonian velocity is larger. In both cases, the greater deviation from the Newtonian profile happens at a distance $\eta \approx 2$ from the wall. In Figs. 2(e) and 2(f), we see that the effect of increasing $\left|K_{0}\right|$ for stagnation point flow $(\gamma=1)$ is the opposite of the flat plate case. Further investigations on the effects of elasticity on the mean flow are given by Cracco. ${ }^{31}$

Note that the non-Newtonian parameter $K_{0}$ in Fig. 2 has been chosen to be large enough to be able to distinguish clearly the nonNewtonian effects on the mean flow. However, as already mentioned, we need $\left|K_{0}\right| \ll 1$ for the boundary layer theory to be valid.

\section{MODAL ANALYSIS}

For the purpose of the stability analysis, we scale the velocities with the constant free-stream velocity $U_{e}\left(x_{0}\right)$ and the lengths with the displacement thickness $\delta_{0}$ relative to the fixed location $x_{0}$, defined by Eq. (10). The new dimensionless variables are

$$
\begin{array}{ll}
\mathbf{x}=\frac{\mathbf{x}_{*}}{\delta_{0}}, & t=\frac{U_{e}\left(x_{0}\right) t_{*}}{\delta_{0}}, \\
\mathbf{v}=\frac{\mathbf{v}_{*}}{U_{e}\left(x_{0}\right)}, & p=\frac{p_{*}}{\rho U_{e}\left(x_{0}\right)^{2}} .
\end{array}
$$

In order to perform a local linear stability analysis, we assume the undisturbed flow to be steady and parallel, neglecting the transverse component of the velocity. The velocity of the base flow in the streamwise direction is taken to be $U_{B}(y)$ given by (14), i.e., the solution of the ODE (13) resulting from the boundary layer approximation at the fixed location $x_{0}$, as shown in Sec. IV.

The governing equations (4) and (5) are linearized around the mean flow $\mathbf{U}_{B}=\left(U_{B}, 0,0\right)^{T}$ and written in terms of the disturbance wall-normal velocity $v$ and the disturbance wall-normal vorticity, $\eta=\frac{\partial u}{\partial z}-\frac{\partial w}{\partial x}$. The linear system governing three-dimensional disturbances is obtained after the application of the normal mode form, as follows:

$$
(v, \eta)=(\hat{v}(y), \hat{\eta}(y)) e^{i(\alpha x+\beta z-\omega t)},
$$

where $\alpha$ and $\beta$ are, respectively, the streamwise ( $x$-direction) and spanwise ( $z$-direction) wavenumbers and $\omega$ represents the frequency.

Defining $\mathbf{q}=(\hat{v}, \hat{\eta})^{T}$, the problem to be solved is a linear system of the form

$$
\mathscr{L} \mathbf{q}=\omega \mathscr{M} \mathbf{q}
$$

where $\mathscr{M}$ and $\mathscr{L}$ are linear operators defined as follows:

$$
\begin{gathered}
\mathscr{M}=\left[\begin{array}{cc}
k^{2}-\mathscr{D}^{2}+K_{0}\left(k^{2}-\mathscr{D}^{2}\right)^{2} & 0 \\
0 & 1+K_{0}\left(k^{2}-\mathscr{D}^{2}\right)
\end{array}\right], \\
\mathscr{L}=\left[\begin{array}{cc}
\mathscr{L}_{O S} & \mathscr{L}_{C N} \\
\mathscr{L}_{C} & \mathscr{L}_{S Q}
\end{array}\right],
\end{gathered}
$$


with

$$
\begin{aligned}
\mathscr{L}_{O S}= & \alpha U_{B}\left(k^{2}-\mathscr{D}^{2}\right)+\alpha U_{B}^{\prime \prime}+\frac{1}{i R e_{0}}\left(k^{2}-\mathscr{D}^{2}\right)^{2} \\
& +K_{0}\left(-\alpha U_{B}^{i v}+\alpha k^{4} U_{B}-2 \alpha k^{2} U_{B} \mathscr{D}^{2}+\alpha U_{B} \mathscr{D}^{4}\right), \\
\mathscr{L}_{C N}= & K_{0}\left(-\beta k^{2} U_{B}^{\prime}-\beta U_{B}^{\prime \prime \prime}+\beta U_{B}^{\prime} \mathscr{D}^{2}\right), \\
\mathscr{L}_{C}= & \beta U_{B}^{\prime}-K_{0} \beta U_{B}^{\prime \prime \prime}, \\
\mathscr{L}_{S Q}= & \alpha U_{B}+\frac{1}{i R e_{0}}\left(k^{2}-\mathscr{D}^{2}\right)+\alpha K_{0} U_{B}\left(k^{2}-\mathscr{D}^{2}\right),
\end{aligned}
$$

where $\mathscr{D}$ denotes the derivative with respect to $y$ and $k^{2}=\alpha^{2}+\beta^{2}$.

We can see that in the Newtonian case, when $K_{0}=0$, the equation for $\hat{v}$ does not involve the wall-normal vorticity $\hat{\eta}$ and reduces to the well-known Orr-Sommerfeld equation when $\beta=0$. Instead, the equation for $\hat{\eta}$, also known as Squire's equation, is driven by solutions to the Orr-Sommerfeld equation through the forcing term $\beta U^{\prime} \hat{v}$. In the Newtonian case, this term is responsible for an algebraic growth of energy and is referred to as the vortex tilting term. We observe that, for a non-zero non-Newtonian parameter $K_{0}$ and a non-zero spanwise wavenumber $\beta$, system (17) is fully coupled due to the presence of a purely non-Newtonian coupling operator, $\mathscr{L}_{\text {CN }}$. tions

The system of equations (17) is subject to the boundary condi-

$$
\hat{v}=\mathscr{D} \hat{v}=\hat{\eta}=0 \quad \text { at } \quad y=0 \quad \text { and } \quad y \rightarrow \infty .
$$

The conditions at $y=0$ are due to the no-slip and no-penetration at the rigid wall. The conditions at infinity derive from assuming that the disturbances tend to zero far from the surface of the plate.

The linear stability equations (17) are solved using a Chebyshev collocation method. ${ }^{33}$ The semi-infinite domain $y \in[0, \infty)$ is mapped into the finite interval $\xi \in[-1,1]$ by means of the algebraic transformation

$$
\xi=\frac{y-2}{y+2}
$$

All the numerical results are validated in the Newtonian limiting case by comparing with results in the literature. ${ }^{30,34}$

\section{A. Growth rates}

At first, we focus on two-dimensional disturbances where $\beta=0$ and we solve the equation in the first row of system (17), which consists of a modified Orr-Sommerfeld equation. ${ }^{24}$ In Fig. 3, the eigenvalues resulting from the linear temporal analysis of the flow over a flat plate $(\gamma=0)$ are displayed. In Fig. 3(a), we compare the eigenspectrum for the second order model with $K=K_{0} C^{2}=0.03$ with eigenvalues obtained in the Newtonian case. The choice of Reynolds number $R e=R e_{0} / C=580$ and wavenumber $\alpha^{*}=\alpha / C=0.179[C$ defined by (11)] generates an unstable mode (i.e., $c_{i}>0$ ) in the Newtonian case, known as a Tollmien-Schlichting wave. We can see the stabilizing effect of elasticity that moves the unstable mode into the lower half-plane. Thus, the flow is temporally stable for the second order model for this choice of wavenumber, elasticity, and Reynolds numbers. In Fig. 3(b), we compare the eigenvalues for the model with $K=-0.03$ with the Newtonian eigenvalues for the same values of Reynolds number and wavenumber. We observe that in this case, elasticity is destabilizing since it pushes the unstable eigenvalues forward into the positive half-plane. We also note that the structure of the rest of the spectrum is different for the non-Newtonian models depending on the sign of $K_{0}$.

Considering the flat plate configuration $(\gamma=0)$, Fig. 4(a) shows the temporal growth rate $\omega_{i}^{*}=\omega_{i} / C$ as a function of $\alpha^{*}$. We note that when $K=0.01$, the maximum growth rate reduces dramatically from $\omega_{i}^{*} \approx 1.8 \times 10^{-3}$ to about $10^{-3}$. Instead, when $K=-0.01$, the maximum growth rate increases to almost $3 \times 10^{-3}$. In general, decreasing $K$ extends the range of positive rates to shorter waves. Figure 4 (b) shows the spatial growth rate $-\alpha_{i}^{*}$ as a function of frequency $\omega^{*}$. Again, we observe the marked stabilizing effect of elasticity in terms of growth rate reduction for $K=0.01$. We observe that, for $K=-0.05$, the maximum growth rate increases, but not so dramatically. We also note that for some wavenumbers $\alpha^{*}$, the growth rate is actually smaller in the non-Newtonian case. The nonNewtonian effects in both models move the maximum to longer waves.

Since the spatial stability properties seem to reflect the temporal characteristics we choose to focus on the temporal problem. ${ }^{31}$ Figure 5 shows the temporal growth rates in the Newtonian and nonNewtonian cases for different values of $\gamma$. In each case, we observe a reduction in temporal growth rate of the Tollmien-Schlichting

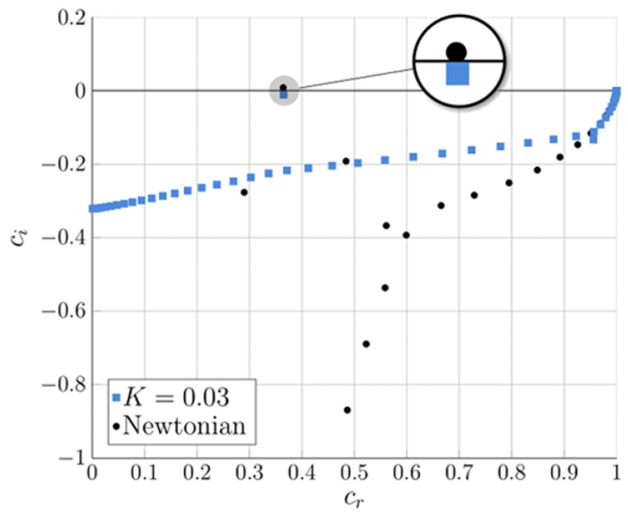

(a)

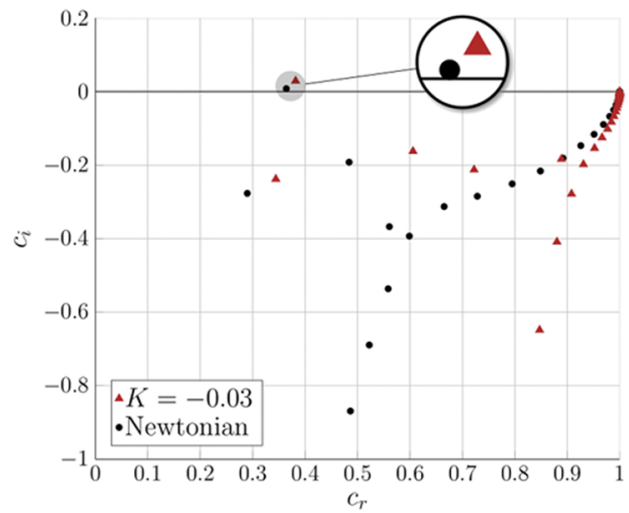

(b)
FIG. 3. Comparison between Newtonian and non-Newtonian eigenspectrum for the temporal problem for flow over a flat plate $(\gamma=0)$ with $\alpha^{*}=0.179, \operatorname{Re}=580$ and (a) $K=0.03$, (b) $K=-0.03$. The least damped eigenvalues are those in the gray circle. 


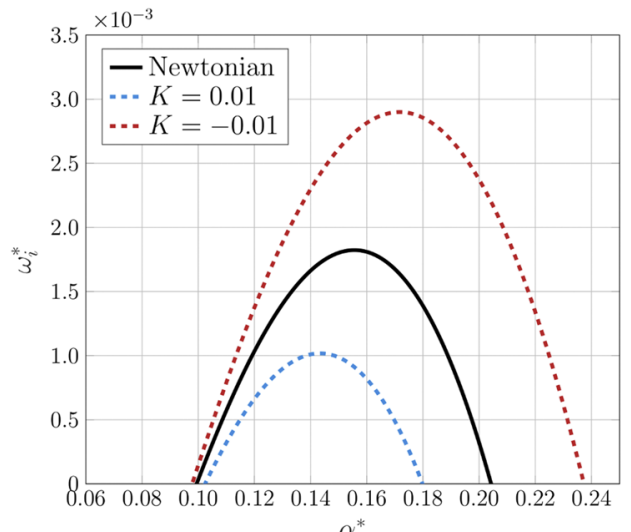

(a)

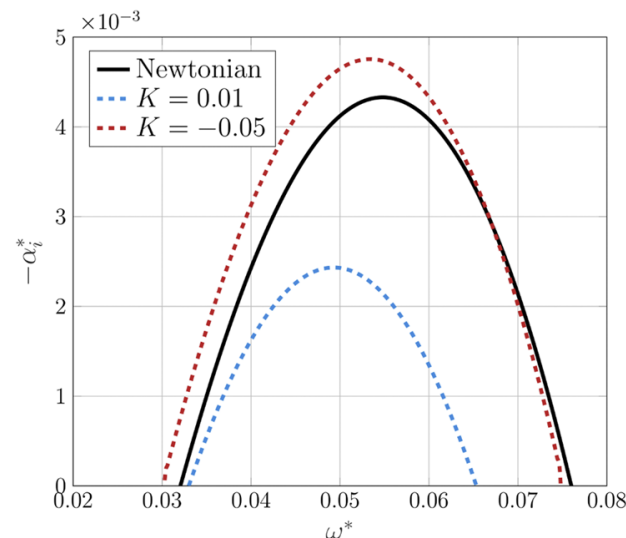

(b)
FIG. 4. Temporal (a) and spatial (b) growth rates for flow over a flat plate $(\gamma$ $=0)$ and $R e=580$. Newtonian and nonNewtonian cases with (a) $K= \pm 0.01$ and (b) $K=0.01,-0.05$. waves due to elasticity for $K>0$ and an increase in growth rate for $K<0$.

\section{B. Neutral stability curves}

Temporal neutral stability curves define the region in the $R e_{0}-\alpha$ plane where exponentially growing modes exist and where they do not. The region inside the curves represents instability, while the region outside corresponds to stability.

In order to plot neutral stability curves, we need to take into account that both $R e_{0}$ and $K_{0}$ depend on the location $x_{0}$. If we decide to perform the stability analysis in which the Reynolds number varies depending on the distance $x_{0}$ from the leading edge to the location where the local stability analysis is performed, then we need

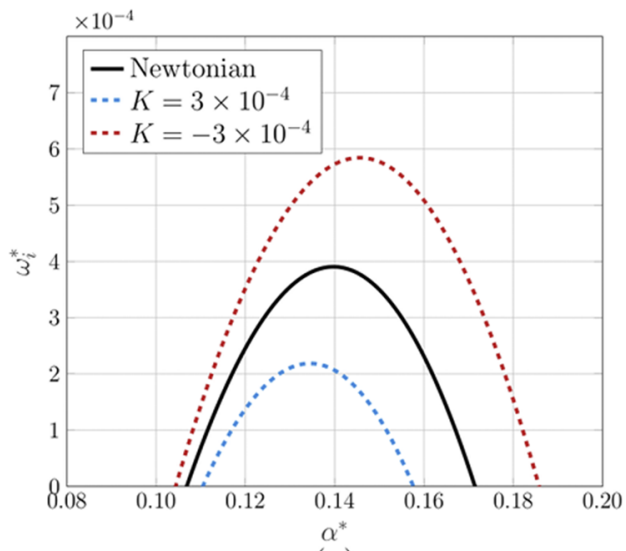

(a)

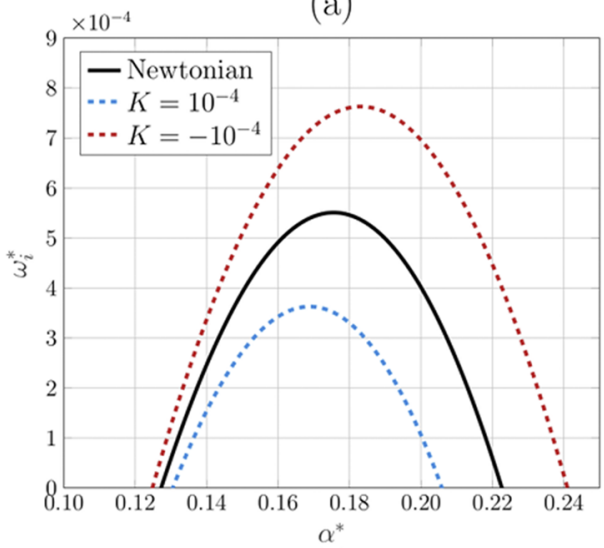

(c)

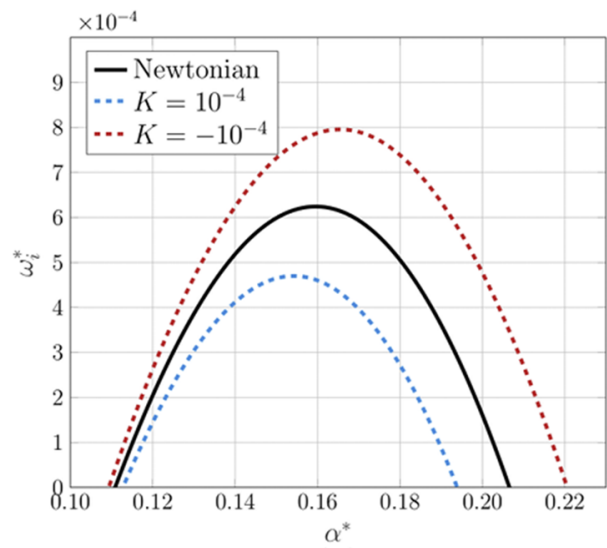

(b)

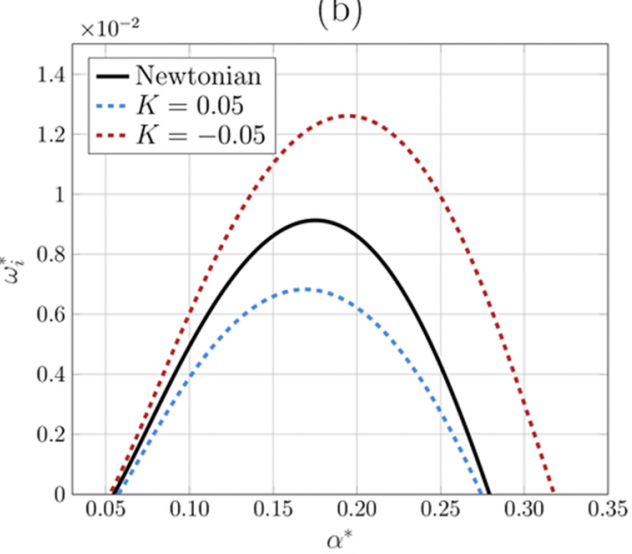

(d)
FIG. 5. Temporal growth rates for a flow past a wedge, Newtonian, and nonNewtonian cases. (a) $\gamma=0.5, R e=$ $10000, K=3 \times 10^{-4}$; (b) $y=1$ (stagnation point), $R e=27000, K=10^{-4}$; (c) $\gamma=1.2, R e=27000, K=10^{-4}$; and (d) $\gamma=-0.14$ (inflection point), $R e=300$, $K=0.05$. 
to write $K_{0}$ in terms of the Reynolds number and the base profile needs to be computed for each value of $R e_{0}$. In the flat plate case $(\gamma=0)$, the non-Newtonian parameter based on the displacement thickness can be rewritten as

$$
K_{0}\left(R e_{0}\right)=\frac{\alpha_{1}}{\rho \delta_{0}^{2}}=\frac{\alpha_{1} a^{2}}{\rho v^{2}} \frac{1}{R e_{0}^{2}} .
$$

Thus, we define the fixed quantity,

$$
\tilde{K}=\frac{\alpha_{1} a^{2}}{\rho v^{2}},
$$

which is independent of $x_{0}$ so that $K_{0}\left(R e_{0}\right)=\tilde{K} / R e_{0}^{2}$.

Figure 6(a) shows a comparison between the neutral stability curves in the Newtonian case and for $\tilde{K}= \pm 10^{3}$ for flow over a flat plate. This clearly shows the stabilizing effect of elasticity in the second order model with $\tilde{K}>0$ in terms of increase of the critical Reynolds number. The non-Newtonian effects in the model with

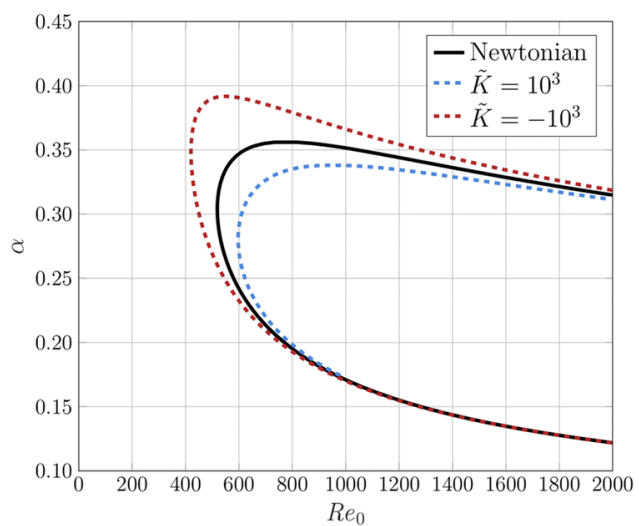

(a)

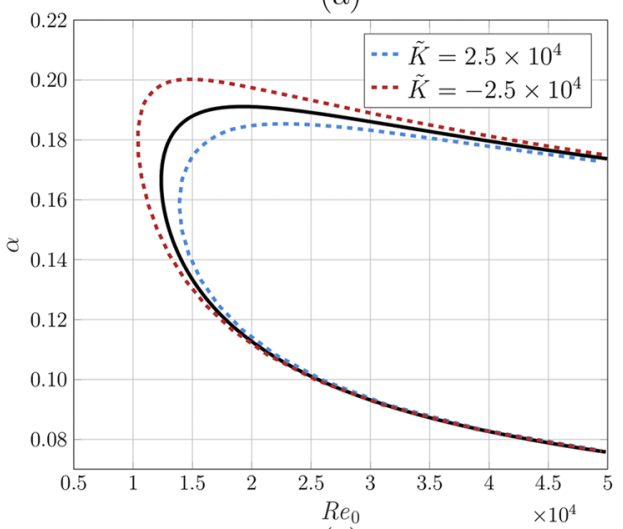

(c)

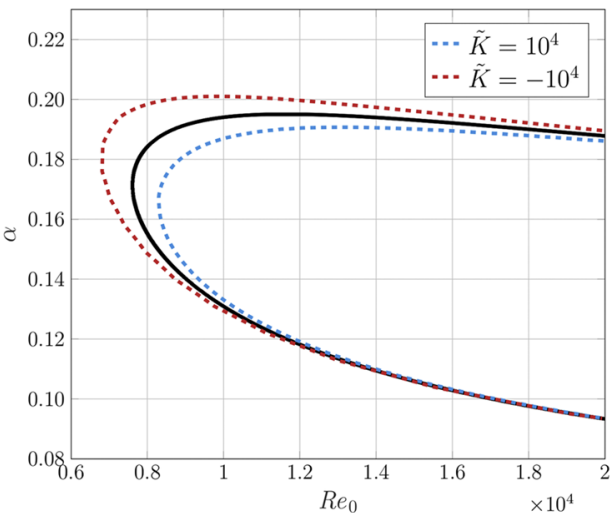

(b)

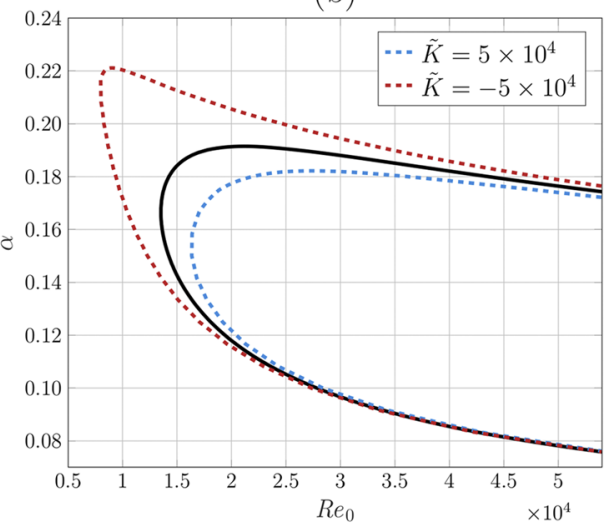

(d)
FIG. 6. Temporal neutral curves in the Newtonian and non-Newtonian cases. (a) $\gamma=0$ (flat plate), $\tilde{K}= \pm 10^{3}$; (b) $\gamma$ $=0.5, \tilde{K}= \pm 10^{4}, x_{0}=1$; (c) $y=1$ (stagnation point), $\tilde{K}= \pm 2.5 \times 10^{4}, x_{0}=1$; (d) $y=1.2, \tilde{K}= \pm 5 \times 10^{4}, x_{0}=1$; and (e) $\gamma$ $=-0.14$ (inflection point), $\tilde{K}= \pm 100 ; x_{0}$ $=1$.

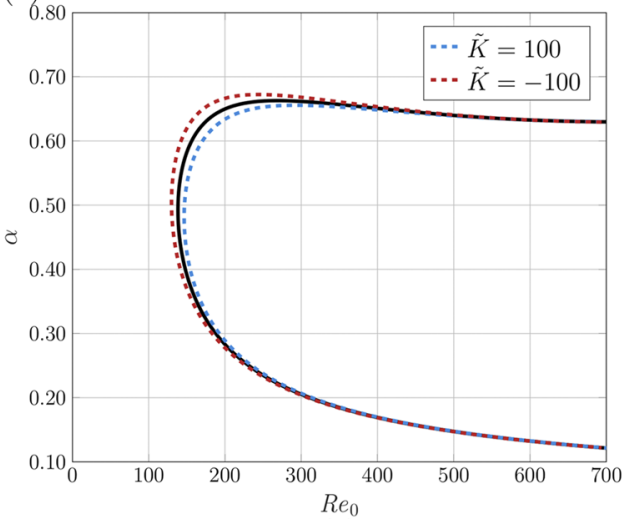

(e) 
$\tilde{K}<0$ promote the onset of instabilities. For high Reynolds numbers, the non-Newtonian neutral curves approach the Newtonian neutral curve. This behavior is expected, since when $R_{0} \rightarrow \infty$, we have $K_{0}$ $\rightarrow 0$.

In the case of a non-zero pressure gradient $(\gamma \neq 0)$, it is not possible to isolate $R e_{0}$ to vary the position $x_{0}$ only through the Reynolds number since we have

$$
K_{0}=\frac{\alpha_{1}}{\rho \delta_{0}^{2}}=\frac{\alpha_{1} a^{2}}{\rho v^{2}} \frac{x_{0}^{2 m}}{R e_{0}^{2}} .
$$

For this reason, we decided to plot the neutral curves in Figs. 6(b)6 (e) by fixing the streamwise position at $x_{0}=1$. In this case, the interpretation must be different, the Reynolds number varies through a variation of the free-stream velocity $U_{e}$. Once again, when $\tilde{K}>0$, elasticity has the effect of reducing the region of two-dimensional instability as shown in Fig. 6 for different angle parameters. When $\tilde{K}<0$, the instability happens at lower Reynolds numbers. Moreover, the neutral curves in the non-Newtonian case approach the Newtonian curves when the Reynolds number increases. It is worth noticing in Fig. 6(e) that, for the flow past a corner $(\gamma=-0.14)$, as the Reynolds number increases, the non-Newtonian curves overlap the Newtonian curve. This means that the inviscid instability, which arises in the presence of an inflectional velocity profile, does not seem to be affected by non-Newtonian effects.

Note that, for different values of $\gamma$, different values of $\tilde{K}$ are chosen in order to ensure that the Weissenberg number, $W i_{0}$, is of order 1 when the Reynolds number is close to critical for the onset of instability. This is to ensure that the boundary layer theory is valid, while the elasticity effects remain significant.

\section{Critical Reynolds numbers}

The critical Reynolds number is defined as the smallest Reynolds number for which there exists an exponentially unstable mode. We calculated the critical wavenumbers, $\alpha_{\mathrm{cr}}$, and Reynolds numbers, $R e_{\mathrm{cr}}$, for different values of $\gamma$, and the results are displayed in Table I. In order to be able to compare the non-Newtonian effect of elasticity for different values of $\gamma$ we choose, as a measure of elasticity, the critical Weissenberg number,

$$
W i_{0, \mathrm{cr}}=K_{0, \mathrm{cr}} R e_{0, \mathrm{cr}},
$$

defined with reference to the Newtonian critical Reynolds number $R e_{0, \mathrm{cr}}$ and the critical elasticity number $K_{0, \mathrm{cr}}=\tilde{K} / R e_{0, \mathrm{cr}}^{2}$. From Table I, we observe that the critical Reynolds numbers for $W i_{0, \mathrm{cr}}>0$ increase for all values of $\gamma$ considered, including the slightly negative value of $\gamma$ that represents a profile with an inflection point. The effect is the opposite for $W i_{0, \mathrm{cr}}<0$, where the critical Reynolds numbers decrease and the instability is anticipated for each value of the angle parameter $\gamma$.

Note that the magnitude of the critical Reynolds number $R e_{0, \mathrm{cr}}$ for the Newtonian case is strongly dependent upon the configuration characterized by $\gamma$. This strong dependence is maintained for the variation found in $R e_{0, \mathrm{cr}}$ when the non-Newtonian effects are introduced in the manner that we have described. For example, with a critical Weissenberg number $W i_{0, \mathrm{cr}}=0.5$, for a flat plate $(\gamma=0)$, the increase or decrease in critical Reynolds number is of order 10 , while for the stagnation point flow $(\gamma=1)$, it is of order $10^{2}$.
TABLE I. Critical Reynolds numbers and critical wavenumbers in the Newtonian and

\begin{tabular}{|c|c|c|c|c|c|}
\hline \multirow[b]{2}{*}{$W i_{0, \mathrm{cr}}$} & \multicolumn{2}{|c|}{ Non-Newtonian } & \multirow{2}{*}{$\frac{\text { Newtonian }}{0}$} & \multicolumn{2}{|c|}{ Non-Newtonian } \\
\hline & -1 & -0.5 & & 0.5 & 1 \\
\hline$\gamma$ & $R e_{0, \mathrm{cr}}$ & $R e_{0, \mathrm{cr}}$ & $R e_{0, \mathrm{cr}}$ & $R e_{0, \mathrm{cr}}$ & $\operatorname{Re}_{0, \mathrm{cr}}$ \\
\hline-0.14 & 126.68 & 132.58 & 138.42 & 144.07 & 149.48 \\
\hline 0 & 470.71 & 495.70 & 519.06 & 540.96 & 561.60 \\
\hline 0.5 & 7005.78 & 7324.05 & 7617.06 & 7890.03 & 8146.65 \\
\hline 1 & 11483.50 & 11949.02 & 12380.61 & 12784.75 & 13166.26 \\
\hline 1.2 & 12563.43 & 13064.70 & 13529.76 & 13965.65 & 14377.28 \\
\hline$\gamma$ & $\alpha_{\mathrm{cr}}$ & $\alpha_{\mathrm{cr}}$ & $\alpha_{\mathrm{cr}}$ & $\alpha_{\mathrm{cr}}$ & $\alpha_{\mathrm{cr}}$ \\
\hline-0.14 & 0.5115 & 0.5025 & 0.4920 & 0.4843 & 0.4774 \\
\hline 0 & 0.3231 & 0.3130 & 0.3038 & 0.2965 & 0.2902 \\
\hline 0.5 & 0.1776 & 0.1742 & 0.1713 & 0.1687 & 0.1664 \\
\hline 1 & 0.1722 & 0.1692 & 0.1665 & 0.1642 & 0.1622 \\
\hline 1.2 & 0.1720 & 0.1690 & 0.1665 & 0.1643 & 0.1622 \\
\hline
\end{tabular}
non-Newtonian cases.

\section{3D results}

We solved the three-dimensional eigenvalue problem (17). The results obtained are summarized by displaying the neutral stability curves in the $\alpha-\beta$ plane. A study of three-dimensional disturbances for fluids of second order is required. For parallel Newtonian flow, Squire's theorem justifies the study of two-dimensional instead of three-dimensional disturbances. Squire's theorem states that each three-dimensional mode corresponds to some two-dimensional mode at a lower Reynolds number. Therefore, to determine the critical Reynolds number, it is sufficient to study two-dimensional disturbances for Newtonian fluids. A result similar to Squire's theorem for a fluid of second order cannot be proven. Therefore, an extension to the study of three-dimensional disturbances is necessary.

Figure 7 shows the contour plot of the temporal growth rate $\omega_{i}$ in the Newtonian case for the flat plate $(\gamma=0)$. Figure 7 (a) shows that the choice of a subcritical Reynolds number $\left(R e_{0}=500\right)$ gives a stable flow. In Fig. 7(b), we increase the Reynolds number to $R e_{0}=1000$, and we can see an exponential instability for which $\omega_{i}>0$, appearing at small spanwise wavenumbers. The red asterisk $(*)$ represents the maximum growth rate reached in the $\alpha-\beta$ plane, i.e., $\max _{\alpha, \beta} \omega_{i}$. We can see that, in both cases, the maximum is reached for spanwise independent waves. This confirms Squire's theorem for Newtonian fluids.

Figures $8(a)$ and $8(b)$ show the contour plots of the temporal growth rates $\omega_{i}$ for the model with $K<0$ and for the model with $K$ $>0$, respectively. We can see that when $K>0$, there is a region of exponential instability for small streamwise wavenumbers and for a value of the Reynolds number $\left(R e_{0}=500\right)$ that corresponds to a stable flow in the Newtonian case. In Fig. 8(c), we displayed the growth rates for a fixed and small $\alpha$ and for a fixed value of $\beta$ in Fig. 8(d). We observe how a positive elasticity number $K$ destabilizes spanwise disturbances, while it stabilizes the Tollmien-Schlichting waves. The opposite happens for a negative value of $K$, which decreases the 


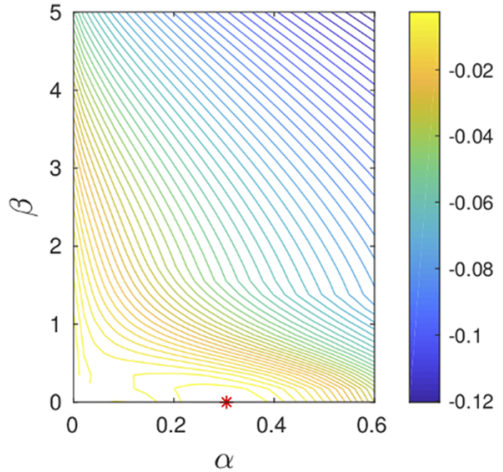

(a)

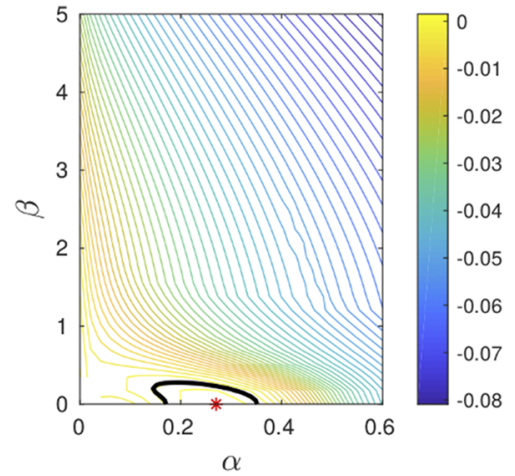

(b)
FIG. 7. Contour plot for the temporal growth rate, $\omega_{i}$ in the Newtonian case $(K=0)$ for the flat plate $(\gamma=0)$. The red asterisk $(*)$ represents $\max _{\alpha \beta} \omega_{i}$. The black line represents the neutral curve. (a) $R e_{0}=500$ and (b) $R e_{0}=1000$. growth rates of mainly streamwise independent waves $(\alpha \approx 0)$ and increases the growth rates of mainly spanwise independent waves $(\beta$ $\approx 0$ ).

Figure 9 shows the results for a Reynolds number of $R e_{0}=1000$. The conclusions are the same, for $K>0$, the Tollmien-Schlichting wave is slightly stabilized while growth rates near the $\alpha=0$ axis become larger. The opposite happens for $K<0$. Figure 10 shows growth rates for the flow past a corner with $\gamma=-0.14$. The results are very similar to those for the flat plate. We do not report results for other values of the angle parameter $\gamma$ since they are in line with the results we have discussed so far.

To the best of our knowledge, the differing effects of elasticity on two-dimensional and three-dimensional disturbances have not been observed in the past for other viscoelastic models.

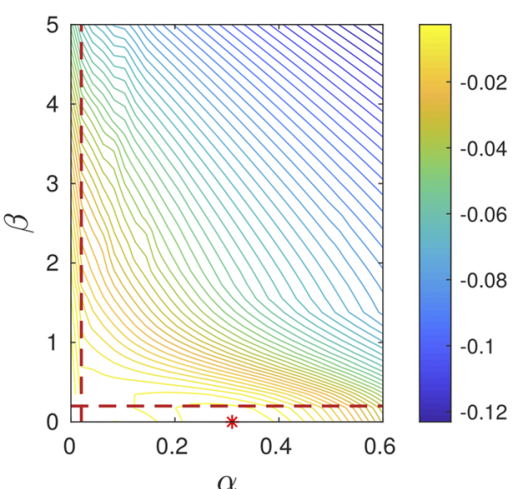

(a)

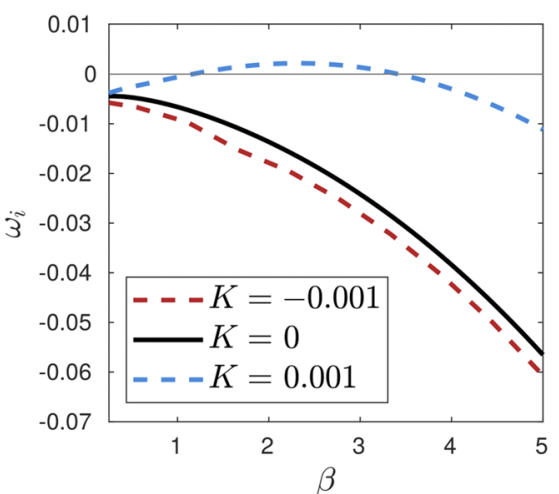

(c)

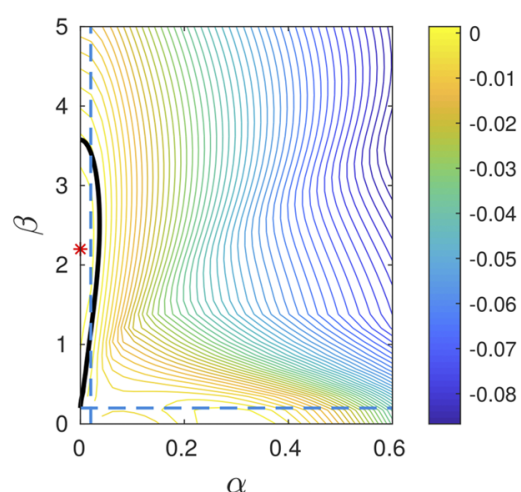

(b)

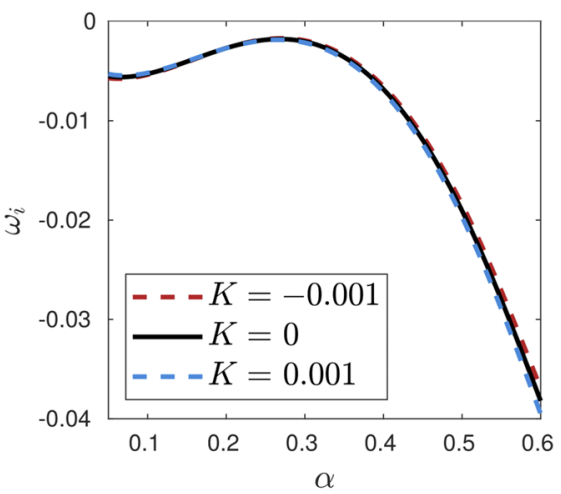

(d)
FIG. 8. [(a) and (b)] Contour plots for $\omega_{1}$ in the non-Newtonian cases for the flat plate $(\gamma=0)$ and $\operatorname{Re}_{0}=500$. The red asterisk $(*)$ represents $\max _{\alpha, \beta} \omega_{i}$. The black line represents the neutral curve. (a) $K=-0.001$ and (b) $K=0.001$. [(c) and (d)] Comparison of Newtonian (solid line) and non-Newtonian (dashed line) temporal growth rates for (c) $\alpha=0.02$ and (d) $\beta=0.2$. 


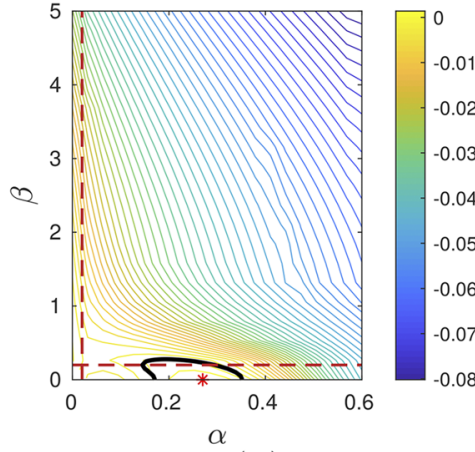

(a)

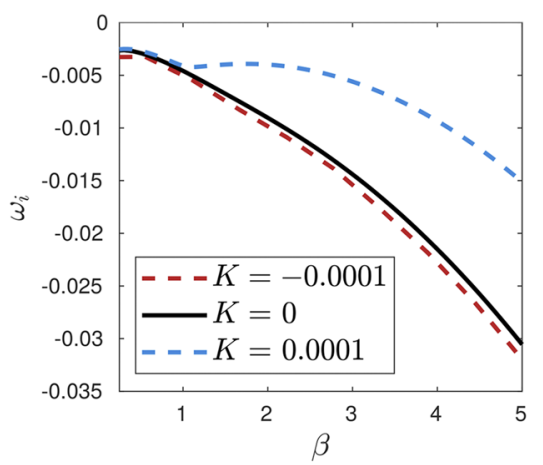

(c)

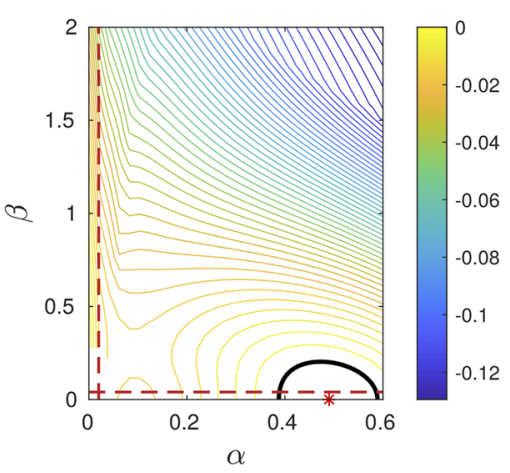

(a)

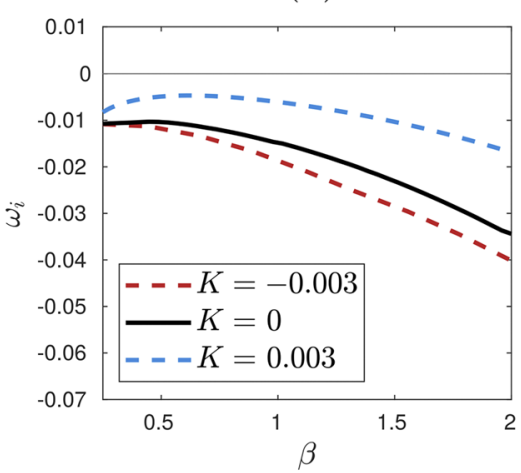

(c)

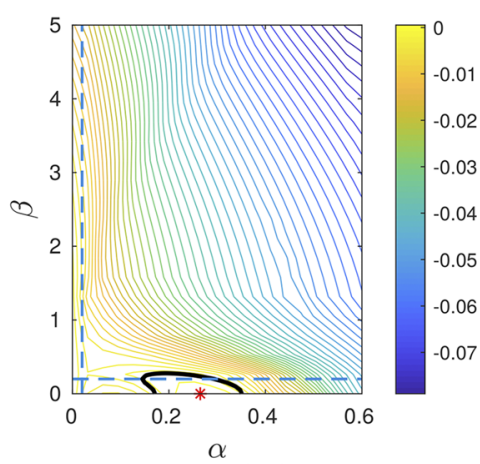

(b)

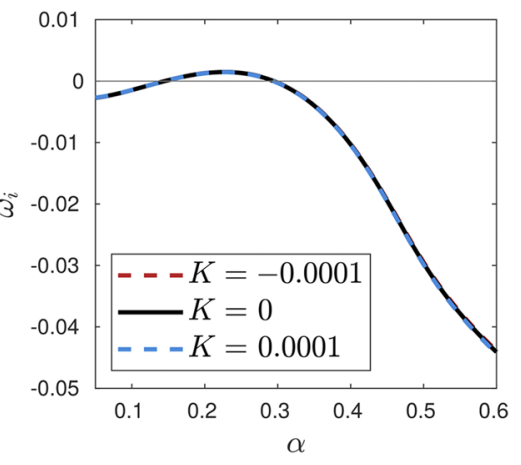

(d)

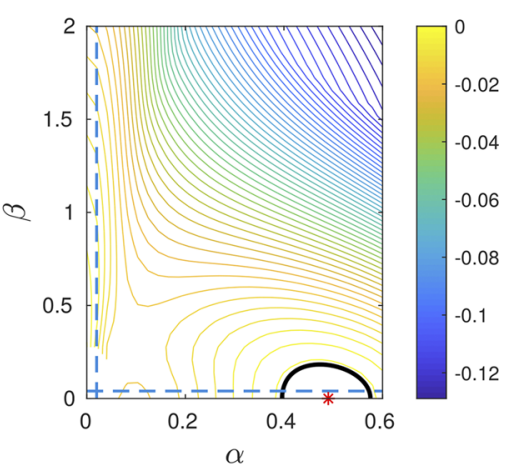

(b)

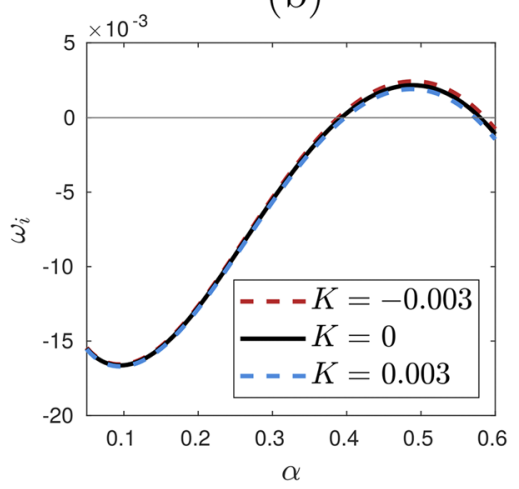

(d)
FIG. 9. [(a) and (b)] Contour plots for $\omega_{i}$ in the nonNewtonian cases for the flat plate $(\gamma=0)$ and $\operatorname{Re}_{0}=1000$. The red asterisk $(*)$ represents $\max _{\alpha, \beta} \omega_{i}$. The black lines represent neutral curves. (a) $K=-0.0001$ and (b) $K=$ 0.0001 . [(c) and (d)] Comparison of Newtonian (solid line) and non-Newtonian (dashed line) temporal growth rates for (c) $\alpha=0.02$ and (d) $\beta=0.2$.
FIG. 10. [(a) and (b)] Contour plots for $\omega_{i}$ in the nonNewtonian cases for the flow past a corner $(\gamma=-0.14)$ and $R e_{0}=150$. The red asterisk (*) represents $\max _{\alpha, \beta} \omega_{i}$. The black lines represent neutral curves. (a) $K=-0.003$ and (b) $K=0.003$. [(c) and (d)] Comparison of Newtonian (solid line) and non-Newtonian (dashed line) temporal growth rates for (c) $\alpha=0.02$ and (d) $\beta=0.04$. 


\section{NON-MODAL ANALYSIS}

In this section, the initial-value problem that drives the development of disturbances is derived for the second order fluid. A formulation based on the initial-value problem enables us to study the behavior of general solutions not only of single eigenmodes. ${ }^{30}$

Brandt $^{19}$ reviewed the main results in bypass transition for non-Newtonian fluids. Zhang et al. ${ }^{27}$ performed the modal and non-modal linear analysis of the inertia-dominated channel flow of viscoelastic fluids modeled by Oldroyd-B and FENE-P closures. The authors observed that both modal and non-modal instabilities are enhanced when the polymer relaxation time is shorter than the instability timescale (i.e., for Weissenberg numbers, $W i \lesssim 1$ ), whereas the flow is more stable in the opposite case. In the subcritical regime, the non-modal amplification of streamwise elongated structures is still the most dangerous energy growth mechanism and is slightly enhanced by the presence of polymers. The lift-up effect is still the dominant instability mechanism also for viscoelastic fluids.

Hoda et al. ${ }^{35}$ studied energy amplification in channel flows of Oldroyd-B fluids from an input-output point of view and found that increasing fluid elasticity through polymer contribution to the viscosity or the elasticity number enhances energy amplification. Once again, the disturbances that are most amplified are streamwiseelongated, with elasticity acting to reduce spanwise length scale.

Some other authors focused on weakly inertial or inertialess flows (e.g., Lieu et al. ${ }^{36}$ and Page and $\mathrm{Zaki}^{37}$ ), while more recently, others studied the secondary instability of streaks for viscoelastic fluids (e.g., Burshtein et al. ${ }^{38}$ ).

After linearization around the mean flow $\mathbf{U}_{B}=\left(U_{B}, 0,0\right)^{T}$, we take the normal mode form for the perturbations. However, unlike in Sec. V, we do not assume an exponential time-dependence. The initial value problem can be written as follows:

$$
\mathscr{M} \frac{\partial \mathbf{q}}{\partial t}=-i \mathscr{L} \mathbf{q}, \quad \text { with } \mathbf{q}=\mathbf{q}_{0} \text { at } t=0,
$$

where $\mathbf{q}(t, y)=(\hat{v}(t, y), \hat{\eta}(t, y))^{T}$. The linear operators $\mathscr{M}, \mathscr{L}$ are defined by Eq. (18). General solutions of the initial-value problem (19) are assumed to belong to the space $\mathbb{S}^{N}$ spanned by a sufficient number $N$ of eigenfunctions, which is defined as follows:

$$
\mathbb{S}^{N}=\operatorname{span}\left\{\tilde{\mathbf{q}}_{1}, \tilde{\mathbf{q}}_{2}, \ldots, \tilde{\mathbf{q}}_{N}\right\},
$$

where $\left\{\tilde{\mathbf{q}}_{j}\right\}_{j}$ are solutions of the eigenvalue problem (17). In other words, $\mathbf{q} \in \mathbb{S}^{N}$ can be expressed as

$$
\mathbf{q}=\sum_{j=1}^{N} k_{j}(t) \tilde{\mathbf{q}}_{j},
$$

where $\left\{k_{j}\right\}_{j}$ are the coefficients of the expansion. This allows us to express the initial value problem (19) as $N$ separated ordinary differential equations for the expansion coefficients, as follows:

$$
k_{j}^{\prime}(t)=-i \omega_{j} k_{j}(t) \quad \text { for } j=1, \ldots, N,
$$

or in a more compact form, i.e.,

$$
\mathbf{k}^{\prime}(t)=-i \Omega \mathbf{k}(t)
$$

where $\mathbf{k}=\left(k_{1}, \ldots, k_{N}\right)^{T}$ and $\Omega=\operatorname{diag}\left\{\omega_{1}, \ldots, \omega_{N}\right\}$. The simplified formulation (21) of the initial-value problem (19) is possible provided that the eigenspectrum is a complete set composed of discrete eigenmodes. For Newtonian fluids, it is known that if the domain is bounded, then the eigenspectrum is discrete, but for unbounded boundary layers, the spectrum is composed of a discrete and a continuous part. Although the discrete approximation differs from the exact representation, the sum of these eigenmodes correctly describes the solutions to the initial-value problem. ${ }^{1}$

For Newtonian fluids, the completeness of the spectrum is proven by Gustavsson. ${ }^{39}$ To the best of our knowledge, the completeness of the spectrum has not been proven yet for second order fluids or non-Newtonian fluids in general. We will not research this further in this paper, and we discretize the continuous spectrum for the second order models, as done by Butler and Farrell. ${ }^{17}$ Therefore, particular attention is paid to ensure that the results are independent of the discretization parameter.

In order to determine the perturbation that grows the most in some sense, we need a way to quantify the growth. The energy norm is taken to be

$$
E(\mathbf{q})=\frac{1}{k^{2}} \int_{0}^{\infty} \mathbf{q}^{H} \mathscr{M} \mathbf{q} d y
$$

where $\mathscr{M}$ is defined by (18a). In order to quantify the transient growth, we define the maximum possible amplification of initial energy density as follows:

$$
G(t, \alpha, \beta)=\max _{\mathbf{q}_{0} \in S^{N} \backslash\{0\}} \frac{\|\mathbf{q}(t)\|_{E}^{2}}{\left\|\mathbf{q}_{0}\right\|_{E}^{2}}=\left\|e^{\mathscr{L}_{1} t}\right\|_{E}^{2},
$$

where $\mathscr{L}_{1}=-i \mathscr{M}^{-1} \mathscr{L}$ and $\mathscr{L}, \mathscr{M}$ are the linear operators given by (18). Fixing the wavenumber vector $(\alpha, \beta)$, the function $G$ represents the envelope of the energy evolution of all the initial perturbations, $\mathbf{q}_{0}$, with unit energy norm. At each moment in time, we maximize over all possible initial conditions. Note that traditional stability analysis focuses attention only on the eigenvalues of $e^{-i \Omega t}$. These do not capture the whole behavior of $G$, which is also determined by the eigenvector matrix $F$ and its inverse. Deducing the behavior of $G$ from the eigenvalue matrix $\Omega$ alone is only valid when the similarity transformation given by $F$ does not alter the norm, that is, when $V$ is unitary and composed of orthogonal eigenvectors. This is the case when $B$ is normal. If this is not the case, $B$ is non-normal and short-time growth of perturbation energy is possible even though the matrix has stable eigenvalues.

In order to compute the exponential norm (23), we use the decomposition (20). Thus, $G$ can be calculated easily as follows:

$$
G(t, \alpha, \beta)=\max _{\mathbf{k}_{0} \in \mathbb{C}^{N} \backslash\{0\}} \frac{\|\mathbf{k}(t)\|_{E}^{2}}{\left\|\mathbf{k}_{0}\right\|_{E}^{2}}=\sigma_{1}^{2}\left(F e^{-i \Omega t} F^{-1}\right),
$$

where $\sigma_{1}$ is the principal singular value of the matrix $B=F e^{-i \Omega t} F^{-1}$. Employing the decomposition (20) provides an easy way to compute the maximum possible amplification $G$, which can be obtained by calculating the singular value decomposition (SVD) of the matrix $B$. 


\section{A. Global optima}

We define the global optimal disturbance as the initial condition, $\mathbf{q}_{0}$, that maximizes the growth over time, i.e.,

$$
G_{\max }(\alpha, \beta)=G\left(t_{\max }, \alpha, \beta\right)=\max _{t \in[0, \infty)} G(t, \alpha, \beta) .
$$

Note that $G_{\max }$ can only be defined when all the eigenvalues are stable. If an unstable mode exists, then $G(t) \rightarrow \infty$ as $t \rightarrow \infty$. We can also define the largest global growth obtained for any wavenumber vector as follows:

$$
G_{\Gamma}=G_{\max }\left(\alpha_{\Gamma}, \beta_{\Gamma}\right)=\max _{\alpha, \beta} G_{\max }(\alpha, \beta) .
$$

The latter depends only on the base flow conditions and $R e$.

The results obtained have been validated by comparing with those found in the literature for Newtonian fluids. For this purpose, we refer to the book by Schmid and Henningson ${ }^{30}$ and the paper by Corbett and Bottaro. ${ }^{40}$ Figure 11 shows the contour plot of $G_{\max }$ defined by (24) for the flat plate $(\gamma=0)$. The black line represents the neutral stability curve inside which an exponentially growing mode exists and where the maximum possible amplification is not defined or can be thought of as infinite. The Newtonian results in Fig. 11(a) are in agreement with the literature. ${ }^{30,41}$ The largest global optimal growth defined by (25) is $G_{\Gamma}=1515.6$ reached at time $t=782$ for $\alpha_{\Gamma}=0, \beta_{\Gamma}=0.65$, as calculated by Corbett and Bottaro. ${ }^{40}$

Figures 11(b) and 11(c) show the contour plot for $K=10^{-4}$ and $K=-10^{-4}$, respectively. These non-Newtonian parameters have been chosen as an example to show the non-Newtonian effects. We can see that the largest amplification of energy is still reached for streamwise independent disturbances, as in the Newtonian case. However, when $K>0$, the amplification of energy is generally larger, and when $K<0$, the amplification of energy is smaller than in the Newtonian case. Figure 12 shows the contour plot of $G_{\max }$ for the flow past a wedge $(\gamma=0.5)$. The non-Newtonian effects on the transient growth are qualitatively similar to the flat plate case. Figure 13(a) displays the ratio of non-Newtonian $G_{\max }$ to Newtonian $G_{\max }$ for a fixed spanwise wavenumber $\beta=0.6$ and varying Weissenberg number $W i_{0}$. We can observe that the non-Newtonian terms mostly affect streamwise independent disturbances, i.e., for $\alpha=0$. In Fig. 13(b), we can see that for $K>0$, the global optima happen at larger times than in the Newtonian case, while for $K<0$, the global optima happen at shorter times.

In Table II, we report the largest global optima $G_{\Gamma}$ defined in (25). For these calculations, we choose the momentum thickness scaling, following Corbett and Bottaro. ${ }^{40}$ The reason is that when scaled using the momentum thickness, the spanwise wavenumber

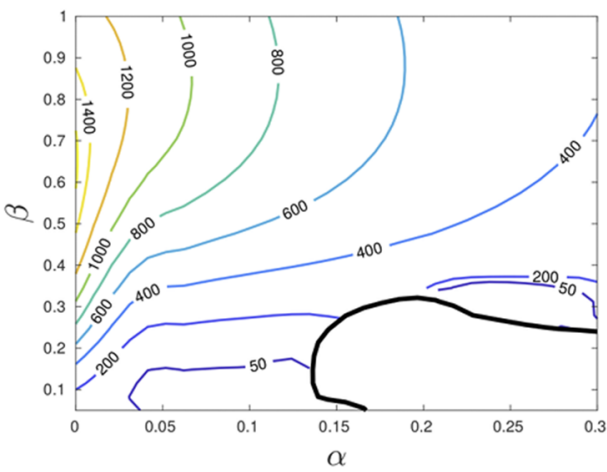

(a)

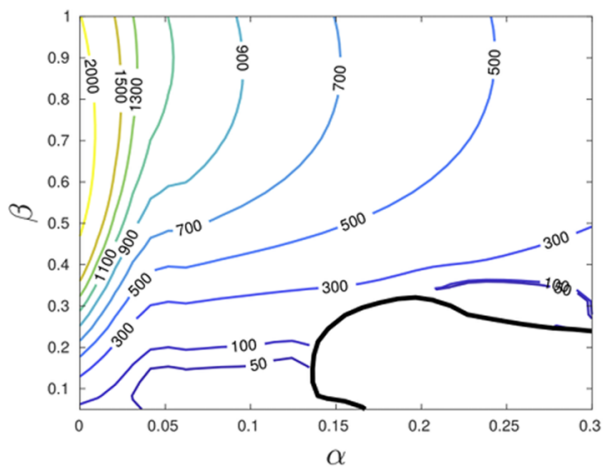

(b)

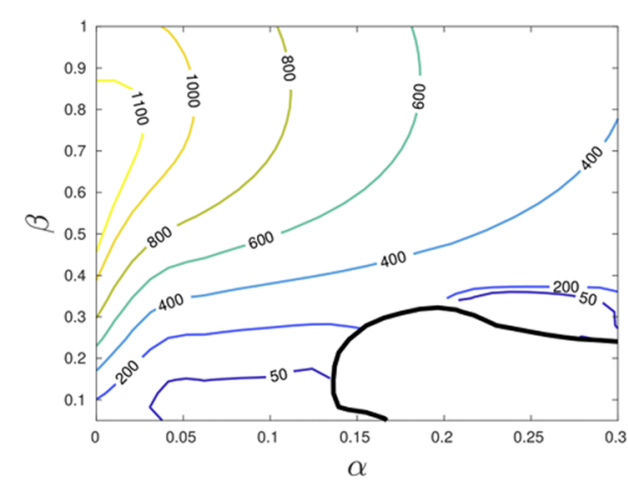

(c)
FIG. 11. Contour plot of $G_{\max }$ for $\gamma$ $=0$ (flat plate) and $R e_{0}=1000$. The black line indicates where an exponentially unstable mode exists. (a) $K=0$, (b) $K=10^{-4}$, and $(c) K=-10^{-4}$. 


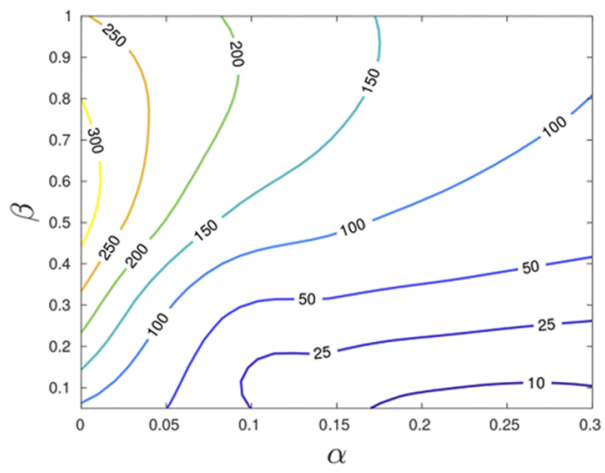

(a)

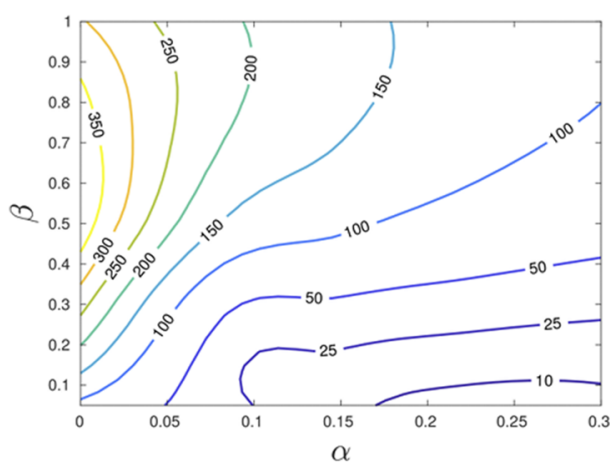

(b)

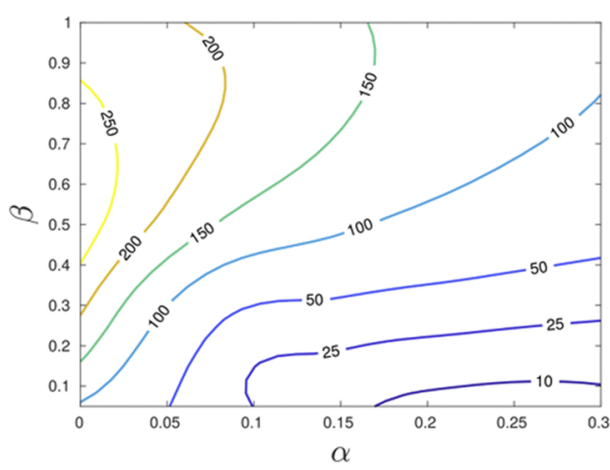

(c)
FIG. 12. Contour plot of $G_{\max }$ for $\gamma=0.5$ and $R e_{0}=500$. (a) $K=0$, (b) $K=10^{-4}$, and (c) $K=-10^{-4}$. at which the largest global optimum is reached is independent of the mean flow conditions. Moreover, momentum thickness scaling accounts for the variation in $t_{\Gamma}$ (the time in which the optimal disturbance reaches its maximum) resulting from differences in the base flow.

We choose to scale the lengths with the momentum thickness $\theta_{0}$ relative to the fixed streamwise location $x_{0}$, which is defined as follows:

$$
\theta_{0}=\theta_{\text {Newt }} \delta\left(x_{0}\right),
$$

where $\delta$ is defined by Eq. (8) and $\theta_{\text {Newt }}$ is the constant,

$$
\theta_{\text {Newt }}=\int_{0}^{\infty}\left(f_{\text {Newt }}^{\prime}\left(1-f_{\text {Newt }}^{\prime}\right)\right) d \eta \text {, }
$$

calculated in the Newtonian case. We introduce Reynolds and Weissenberg numbers based on $\theta_{0}$ as follows:

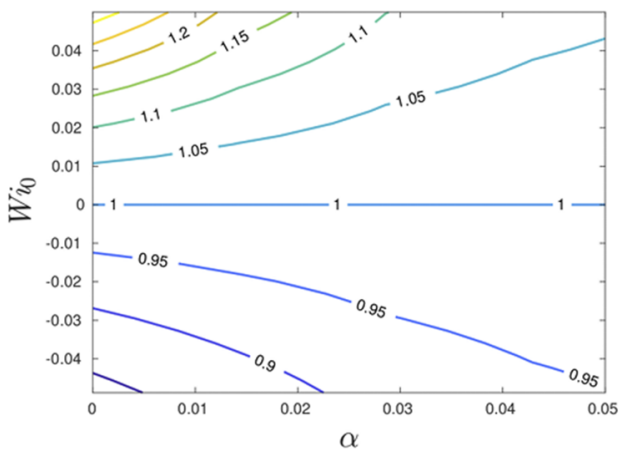

(a)

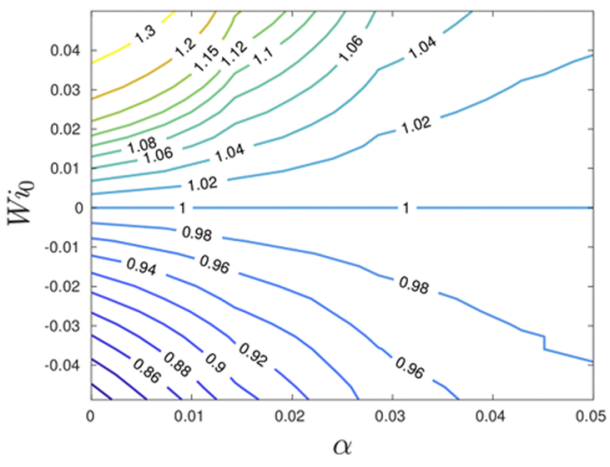

(b)
FIG. 13. Ratio of non-Newtonian to Newtonian maximum possible amplification for the flat plate $\gamma=0$ and $R e_{0}=$ $500, \beta=0.6$. (a) $G_{\max } / G_{\max , \text { Newt }}$ and (b) $t_{\max } / t_{\text {max,Newt. }}$ 
TABLE II. Largest global optima for $R e_{\theta}=166$ and $R e_{\theta}=385$. The asterisk $\left(^{*}\right)$ indicates where an exponentially unstable mode exists and $G_{\Gamma}$ is calculated excluding the TS wave. The missing values indicate where an exponential unstable mode also exists as $\beta \rightarrow 0$.

\begin{tabular}{|c|c|c|c|c|c|c|c|}
\hline \multirow[b]{2}{*}{$\gamma$} & \multicolumn{4}{|c|}{$R e_{\theta}=166$} & \multicolumn{3}{|c|}{$R e_{\theta}=385$} \\
\hline & $W i_{\theta}$ & $\beta_{\theta}$ & $t_{\theta}$ & $G_{\Gamma}$ & $\beta_{\theta}$ & $t_{\theta}$ & $G_{\Gamma}$ \\
\hline \multirow{5}{*}{-0.14} & -0.10 & 0.2390 & 802.03 & $357.49^{*}$ & 0.2347 & 1629.56 & $1674.25^{*}$ \\
\hline & -0.05 & 0.2410 & 856.30 & $380.72^{*}$ & 0.2386 & 1827.99 & $1888.35^{*}$ \\
\hline & 0 & 0.2432 & 926.30 & $408.82^{*}$ & 0.2432 & 2151.88 & $2202.45^{*}$ \\
\hline & 0.05 & 0.2457 & 1021.51 & $444.12^{*}$ & 0.2494 & 2856.03 & $2749.60^{*}$ \\
\hline & 0.10 & 0.2485 & 1165.67 & $491.16^{*}$ & $\ldots$ & $\ldots$ & $\ldots$ \\
\hline \multirow{5}{*}{-0.07} & -0.10 & 0.2452 & 768.81 & $283.86^{*}$ & 0.2414 & 1562.67 & $1332.59^{*}$ \\
\hline & -0.05 & 0.2470 & 819.99 & $301.87^{*}$ & 0.2448 & 1750.79 & $1498.67^{*}$ \\
\hline & 0 & 0.2489 & 885.33 & $323.59^{*}$ & 0.2489 & 2057.06 & $1742.94^{*}$ \\
\hline & 0.05 & 0.2510 & 973.27 & $350.59^{*}$ & 0.2542 & 2693.02 & $2157.79^{*}$ \\
\hline & 0.10 & 0.2533 & 1100.95 & $385.74^{*}$ & $\cdots$ & $\cdots$ & $\cdots$ \\
\hline \multirow{5}{*}{0} & -0.10 & 0.2475 & 758.86 & 247.29 & 0.2438 & 1544.18 & $1162.12^{*}$ \\
\hline & -0.05 & 0.2491 & 808.67 & 262.74 & 0.2470 & 1730.97 & $1307.12^{*}$ \\
\hline & 0 & 0.2508 & 872.23 & 281.42 & 0.2509 & 2026.73 & $1515.60^{*}$ \\
\hline & 0.05 & 0.2528 & 956.55 & 304.52 & 0.2557 & 2617.47 & $1862.12^{*}$ \\
\hline & 0.10 & 0.2550 & 1075.89 & 334.05 & 0.2649 & 5467.14 & $2771.06^{*}$ \\
\hline \multirow{5}{*}{0.5} & -0.10 & 0.2479 & 765.91 & 168.13 & 0.2446 & 1568.41 & 792.59 \\
\hline & -0.05 & 0.2495 & 812.86 & 178.30 & 0.2476 & 1750.22 & 889.17 \\
\hline & 0 & 0.2512 & 871.08 & 190.36 & 0.2513 & 2024.78 & 1024.65 \\
\hline & 0.05 & 0.2531 & 945.42 & 204.99 & 0.2561 & 2520.51 & 1238.61 \\
\hline & 0.10 & 0.2552 & 1045.30 & 223.25 & 0.2650 & 3948.30 & 1688.11 \\
\hline \multirow{5}{*}{1} & -0.10 & 0.2471 & 774.23 & 147.29 & 0.2436 & 1590.90 & 694.91 \\
\hline & -0.05 & 0.2487 & 820.22 & 156.10 & 0.2467 & 1769.96 & 778.84 \\
\hline & 0 & 0.2504 & 876.65 & 166.49 & 0.2505 & 2037.67 & 895.93 \\
\hline & 0.05 & 0.2524 & 947.52 & 179.00 & 0.2556 & 2504.65 & 1078.00 \\
\hline & 0.10 & 0.2547 & 1041.05 & 194.51 & 0.2651 & 3701.44 & 1440.19 \\
\hline \multirow{5}{*}{1.2} & -0.10 & 0.2469 & 775.71 & 142.60 & 0.2434 & 1594.62 & 672.67 \\
\hline & -0.05 & 0.2485 & 821.55 & 151.13 & 0.2465 & 1773.57 & 753.97 \\
\hline & 0 & 0.2503 & 877.53 & 161.17 & 0.2504 & 2040.22 & 867.26 \\
\hline & 0.05 & 0.2523 & 947.83 & 173.25 & 0.2556 & 2501.90 & 1043.04 \\
\hline & 0.10 & 0.2547 & 1039.94 & 188.21 & 0.2654 & 3662.17 & 1390.06 \\
\hline
\end{tabular}

$$
\operatorname{Re}_{\theta}=\frac{U_{e}\left(x_{0}\right) \theta_{0}}{v}, \quad W i_{\theta}=\frac{\alpha_{1} U_{e}\left(x_{0}\right)}{\mu \theta_{0}}
$$

Note that the following relations hold:

$$
R e_{0}=H R e_{\theta}, \quad W i_{0}=\frac{W i_{\theta}}{H},
$$

where $H=C / \theta_{\text {Newt }}$ is the shape factor defined as the ratio between displacement and momentum thickness, calculated in the Newtonian case. In Table II, we present the results obtained for Reynolds numbers $R e_{\theta}=166$ and $R e_{\theta}=385$. These Reynolds numbers have been chosen to compare the results with the ones obtained by
Corbett and Bottaro. ${ }^{40}$ Specifically, $R e_{\theta}=385$ corresponds to the Reynolds number based on the displacement thickness $R e_{0} \approx 1000$ for the flat plate case.

For all the flows considered, the largest global optimum is reached for streamwise-independent waves, i.e., $\alpha_{\Gamma}=0$. We can see that, in the Newtonian case, when scaled with $\theta_{0}$, the spanwise wavenumber for $G_{\Gamma}$ appears to be independent of the mean flow condition characterized by $\gamma$ and $\beta_{\theta} \approx 1 / 4$. Note that, in the Newtonian case, the moment in time at which the largest global optimum is reached is about the same for all the positive angle parameters considered, $t_{\theta} \approx 880$.

For flow past a corner $(\gamma=-0.14)$, the maximum is reached at a larger time $t_{\theta} \approx 927$. We observe how, for all the angle parameters 
considered, the spanwise wavenumber $\beta_{\theta}$, the time $t_{\theta}$, and the largest possible amplification $G_{\Gamma}$ decrease when the model with $W i_{\theta}<0$ is selected and increase when the model with $W i_{\theta}>0$ is selected. Moreover, $\beta_{\theta}$ appears to change approximately linearly with the Weissenberg number based on the momentum thickness. A Weissenberg number $W i_{0}= \pm 0.05$ produces a change in $\beta_{\theta}$ of about $1 \%$ and $W i_{0}= \pm 0.1$ produces a change of about $2 \%$. This linear dependence on the Weissenberg number manifests also on the time $t_{\theta}$ and on the largest transient growth $G_{\Gamma}$.

\section{B. Optimal perturbations}

We can determine the initial condition that reaches the maximum possible amplification at a given time $t_{0}$ by using the singular value decomposition (SVD) of the matrix $B=F e^{-i t_{0} \Omega} F^{-1}$. The initial condition that reaches the global optimum $G_{\max }$ at $t=t_{\max }$ defined by (24) is referred to as optimal disturbance.

Figure 14 shows a comparison between optimal disturbances in the Newtonian and non-Newtonian cases for the stagnation point flow $(\gamma=1)$ and a Reynolds number $R e_{0}=500$. We choose a wavenumber vector $(\alpha, \beta)=(0,0.6)$, which is close to the global optima. In Figs. 14(a)-14(c), $|\mathbf{u}|$ has been scaled such that $\max \left(\left|v_{0, \text { Newt }}\right|\right)=1$, and in Figs. 14(b)-14(d), $|\mathbf{u}|$ has been scaled such that $\max \left(\left|v_{\text {max }, \text { Newt }}\right|\right)=1$.

We see that the optimal disturbances, in the non-Newtonian cases, have the same structure of streamwise-oriented vortices as in the Newtonian case. From Figs. 14(a)-14(c), we observe that the initial streamwise velocity $\left|u_{0}\right|$ is two orders of magnitude smaller than the cross-flow components, $\left|v_{0}\right|,\left|w_{0}\right|$. Figures 14(b)-14(d) show the evolved state of the optimal disturbances at $t=t_{\max }$. The shape of the initial vortex is still present, although it has diffused outward away from the wall.

At $t=t_{\max }$, the streamwise velocity $\left|u_{\max }\right|$ is one order of magnitude larger than the cross-flow velocities, which indicates the presence of streaks. From Figs. 14(a) and 14(b), we see that when $K>0$, the vortices are more diffused away from the wall, whereas when $K<0$, the vortices are closer to the wall. Figures 14(c) and 14(d) show that, for the non-Newtonian fluid with $K=-0.0001$, the initial optimal streamwise velocity is larger than in the Newtonian case and at $t_{\max }$, it grows more than in the Newtonian case. The behavior is the opposite when $K=0.0001$. This is in agreement with the results obtained in Sec. VI A.

In Fig. 15, we plotted the streamwise vortices for $K=-0.0001$ and $R e_{0}=1000$. The solutions plotted are such that $\left\|\mathbf{q}_{0}\right\|_{E}=1$ and

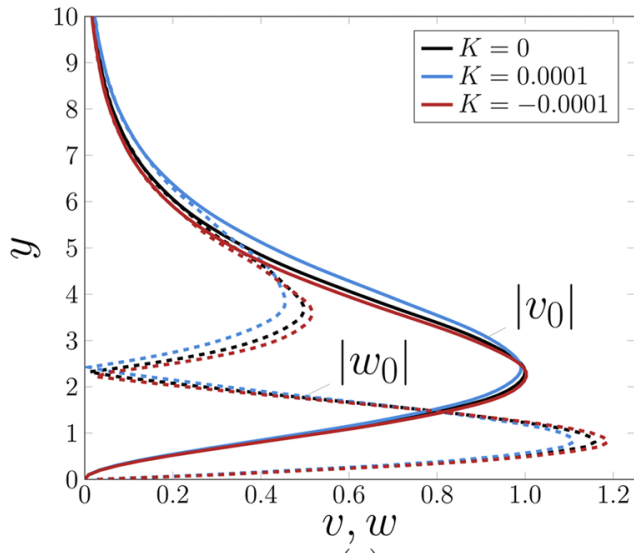

(a)

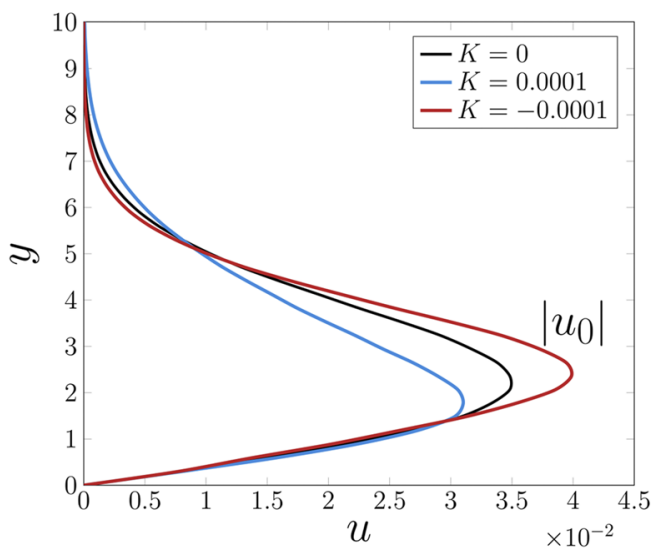

(c)

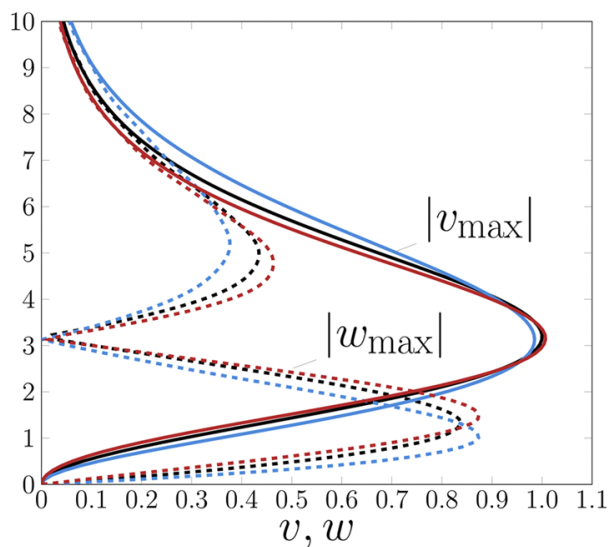

(b)

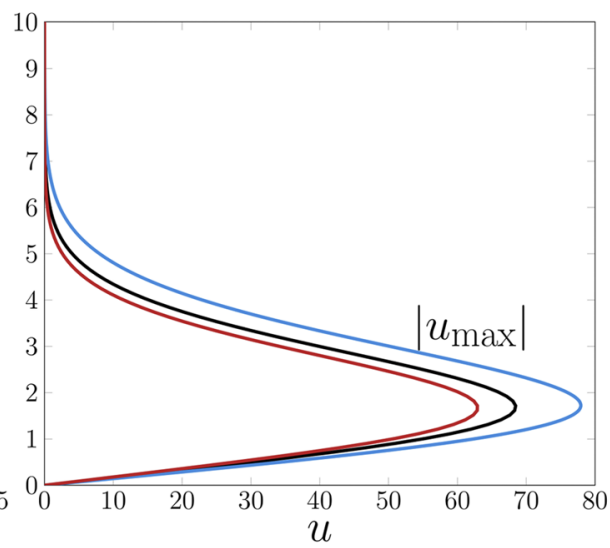

(d)
FIG. 14. Comparison between Newtonian and non-Newtonian optimal disturbances for the stagnation point flow with $\gamma=1, \operatorname{Re}_{0}=500, \alpha=0.6$, and $\beta=0$. (a) Wall-normal, $\left|v_{0}\right|$, and spanwise, $\left|w_{0}\right|$, initial velocities; (b) wall-normal, $\left|v_{\max }\right|$, and spanwise, $\left|w_{\max }\right|$, velocities at $t$ $=t_{\max }$; (c) streamwise, $\left|u_{0}\right|$, initial velocities; and (d) streamwise, $\left|u_{\max }\right|$, streamwise velocity at $t=t_{\max }$. 


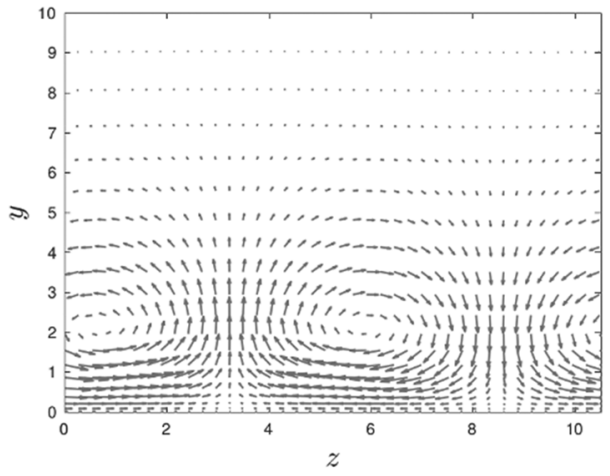

(a)

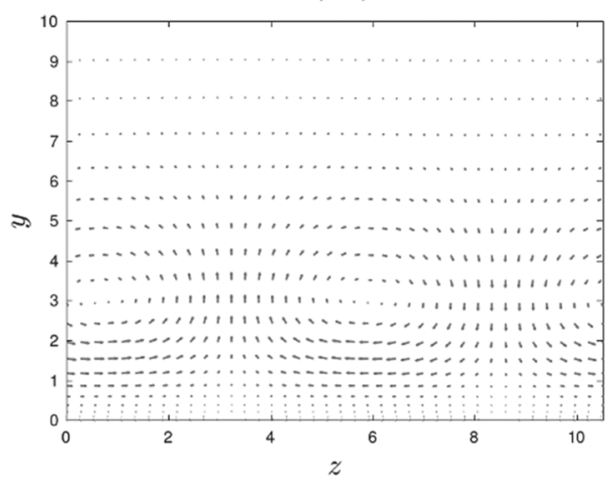

(C)

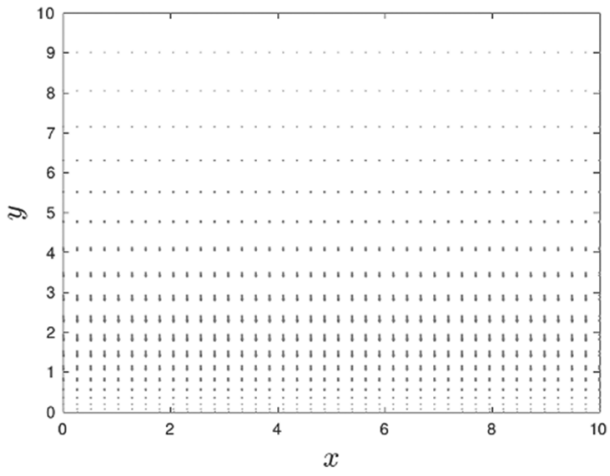

(b)

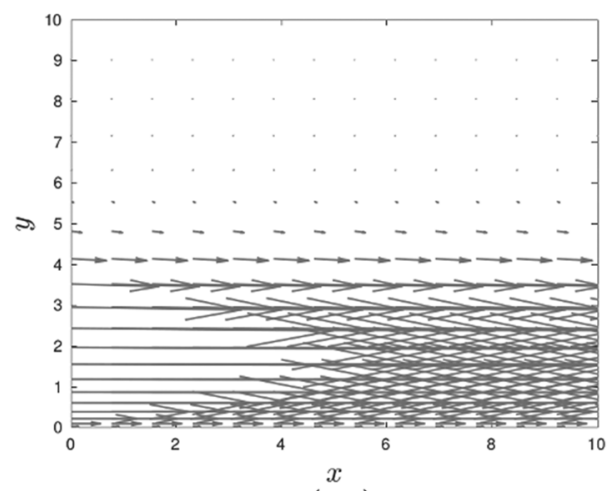

$(d)$
FIG. 15. Optimal disturbance for the stagnation point flow with $\gamma=1, R_{0}$ $=1000, \alpha=0.6$, and $\beta=0$ and a nonNewtonian parameter $K=-0.0001$. [(a) and (b)] disturbance at $t=0$ and [(c) and (d)] disturbance at $t=t_{\max }$. $\left\|\mathbf{q}\left(t_{\max }\right)\right\|_{E}=G_{\max }$. In Figs. 15(a) and 15(c), we can see the streamwise vortices at $t=0$ and $t=t_{\max }$, respectively. From Figs. 15(b) and 15(d), we can see the lift-up effect in action, which transforms streamwise vortices into streamwise streaks.

\section{CONCLUSIONS}

The linear stability analysis of the boundary layer flow of a viscoelastic fluid has been investigated. We applied a boundary layer theory to second order fluids in order to determine the mean flow. As for Newtonian fluids, this approach allowed us to simplify the governing equations. There is no similarity solution to the boundary layer equations with the exception of stagnation point flow. Therefore, a local similarity transformation was applied that yielded a local ODE depending on the streamwise position. We reformulated this equation in order to represent the dependency on the location only through the non-Newtonian parameter $K$ based on the displacement thickness at a fixed streamwise position. The equation obtained is then solved numerically and employed in the linear stability analysis. This approach is consistent with the fact that traditional linear stability analysis is a local analysis.

First, we considered two-dimensional disturbances, namely, disturbances that vary only in the streamwise and wall-normal directions. We solved numerically, using a Chebyshev collocation method, the modified Orr-Sommerfeld equation that governs the evolution of two-dimensional disturbances. The results were presented in terms of growth rates and neutral curves. For all values of the angle parameter $\gamma$, the non-Newtonian terms in the second order model with $K>0$ stabilize the flow with respect to the Newtonian case, while they have the opposite effect for $K<0$. Moreover, we determined the critical Reynolds number, which is the smallest Reynolds number for which there exists an exponentially unstable mode. For $K>0$, there is a stabilizing effect in terms of an increase of the critical Reynolds numbers. The effect is the opposite for $K<0$, where the instability is enhanced. The linear stability results for $K<0$, which is the one that predicts the correct sign of the non-zero normal stress differences, are in qualitative agreement with those obtained by Sureshkumar and Beris ${ }^{26}$ and Zhang ${ }^{27}$ for the Poiseuille flow of other viscoelastic fluids.

When extending the analysis to three-dimensional disturbances, which can also vary in the spanwise direction, the nonNewtonian effects prove to be different. This differing behavior has not been observed before for other viscoelastic models. We showed that a positive elasticity number $K$ destabilizes spanwise disturbances while it stabilizes the two-dimensional TollmienSchlichting waves. The opposite happens for a negative $K$, which decreases the growth rates of mainly streamwise independent waves and increases the growth rates of mainly spanwise independent waves. 
In order to give a complete idea of the linear stability characteristics, the potential transient growth of energy cannot be ignored. To the best of our knowledge, the transient growth of viscoelastic fluids in boundary layers has not been investigated in the past. In this paper, the initial-value problem that drives the development of disturbances is derived for second order fluids. In the Newtonian case, our results are validated against those obtained by Schmid ${ }^{41}$ for Blasius flow and by Corbett and Bottaro ${ }^{40}$ for Falkner-Skan flows. We showed that, for the second order model with $K>0$, an increase in the non-Newtonian parameter $K$ provokes an increase in the maximum transient growth, $G$, while the second order model with $K<0$ has the opposite behavior. The results are qualitatively similar for all values of the angle parameter, $\gamma$. The largest amplification of energy is still reached for streamwise independent disturbances (zero streamwise wavenumber), as in the Newtonian case.

Non-Newtonian terms mostly affect streamwise independent disturbances. For $K>0$, the global optimum, $G_{\max }$, is reached for larger times and shorter waves (larger spanwise wavenumber) than in the Newtonian case. On the contrary, for $K<0$, the global optimum is reached for shorter times and longer waves (smaller spanwise wavenumber).

The second order models are not used in industrial applications, where other constitutive equations are preferred. However, they can represent non-zero normal stress differences, and they give an idea of the stability characteristics of viscoelastic fluids in boundary layers. The natural progression of this work is the investigation of the linear stability properties of rheologically more complex models in boundary layers.

\section{ACKNOWLEDGMENTS}

M.C. would like to acknowledge the Engineering and Physical Sciences Research Council of the United Kingdom (Grant No. $\mathrm{EP} / \mathrm{P} 505453$ ) and Cardiff University for providing financial support for this work.

Part of this work was performed using the computational facilities of the Advanced Research Computing@Cardiff (ARCCA) Division, Cardiff University.

\section{DATA AVAILABILITY}

The data that support the findings of this study are openly available via the Cardiff University data repository at https://doi.org/10.17035/d.2020.0112209117.

\section{REFERENCES}

${ }^{1}$ R. S. Rivlin and J. L. Ericksen, "Stress deformation relations for isotropic materials," J. Ration. Mech. Anal. 4, 323-425 (1955).

${ }^{2}$ R. G. Owens and T. N. Phillips, Computational Rheology (Imperial College Press, 2002).

${ }^{3}$ K. R. Rajagopal, A. S. Gupta, and A. S. Wineman, "On a boundary layer theory for non-Newtonian fluids,” Int. J. Eng. Sci. 18, 875-883 (1980).

${ }^{4}$ K. R. Rajagopal and P. N. Kaloni, "Some remarks on boundary conditions for flows of fluids of the differential type," in Continuum Mechanics and its Applications, edited by G. A. C. Graham and S. K. Malik (Hemisphere Press, 1989).

${ }^{5} \mathrm{~K}$. Rajagopal, "On boundary conditions for fluids of the differential type," in Navier-Stokes Equations and Related Nonlinear Problems, edited by A. Sequeira (Plenum Press, 1995).
${ }^{6}$ D. W. Beard and K. Walters, "Elastico-viscous boundary-layer flows I. Twodimensional flow near a stagnation point," Math. Proc. Cambridge Philos. Soc. 60, 667-674 (1964).

${ }^{7}$ K. R. Rajagopal, A. S. Gupta, and T. Y. Na, “A note on the Falkner-Skan flows of a non-Newtonian fluid,” Int. J. Non-Linear Mech. 18, 313-320 (1983).

${ }^{8}$ V. K. Garg and K. R. Rajagopal, "Stagnation point flow of a non-Newtonian fluid," Mech. Res. Commun. 17, 415-421 (1990).

${ }^{9}$ V. K. Garg and K. R. Rajagopal, "Flow of a non-Newtonian fluid past a wedge," Acta Mech. 88, 113-123 (1991).

${ }^{10} \mathrm{~K}$. Vajravelu and T. Roper, "Flow and heat transfer in a second grade fluid over a stretching sheet,” Int. J. Non-Linear Mech. 34, 1031-1036 (1999).

${ }^{11} \mathrm{~K}$. Vajravelu and D. Rollins, "Hydromagnetic flow of a second grade fluid over a stretching sheet," Appl. Math. Comput. 148, 783-791 (2004).

${ }^{12}$ C. M. White and M. G. Mungal, "Mechanics and prediction of turbulent drag reduction with polymer additives," Annu. Rev. Fluid Mech. 40, 235-256 (2008).

${ }^{13}$ E. De Angelis, C. M. Casciola, V. S. L’vov, R. Piva, and I. Procaccia, "Drag reduction by polymers in turbulent channel flows: Energy redistribution between invariant empirical modes," Phys. Rev. E 67, 056312 (2003).

${ }^{14}$ E. De Angelis, C. M. Casciola, V. S. L'vov, A. Pomyalov, I. Procaccia, and V. Tiberkevich, "Drag reduction by a linear viscosity profile," Phys. Rev. E 70, 055301 (2004).

${ }^{15} \mathrm{~L}$. Xi, "Turbulent drag reduction by polymer additives: Fundamentals and recent advances," Phys. Fluids 31, 121302 (2019).

${ }^{16}$ L. N. Trefethen, A. E. Trefethen, S. C. Reddy, and T. A. Driscoll, "Hydrodynamic stability without eigenvalues,” Science 261, 578-584 (1993).

${ }^{17}$ K. M. Butler and B. F. Farrell, "Three-dimensional optimal perturbations in viscous shear flow,” Phys. Fluids A 4, 1637-1650 (1992).

${ }^{18}$ M. T. Landahl, "A note on an algebraic instability of inviscid parallel shear flows,” J. Fluid Mech. 98, 243-251 (1980).

${ }^{19} \mathrm{~L}$. Brandt, "The lift-up effect: The linear mechanism behind transition and turbulence in shear flows," Eur. J. Mech., B: Fluids 47, 80-96 (2014).

${ }^{20}$ J. E. Dunn and K. R. Rajagopal, "Fluids of differential type: Critical review and thermodynamic analysis,” Int. J. Eng. Sci. 33, 689-729 (1995).

${ }^{21}$ J. E. Dunn and R. L. Fosdick, "Thermodynamics, stability, and boundedness of fluids of complexity 2 and fluids of second grade," Arch. Ration. Mech. Anal. 56, 191-252 (1974).

${ }^{22}$ R. L. Fosdick and K. R. Rajagopal, "Anomalous features in the model of "second order fluids,"” Arch. Ration. Mech. Anal. 70, 145-152 (1979).

${ }^{23}$ K. C. Porteous and M. M. Denn, "Linear stability of plane Poiseuille flow of viscoelastic liquids," Trans. Soc. Rheol, 16, 295 (1972).

${ }^{24}$ D. H. Chun and W. H. Schwarz, "Stability of a plane Poiseuille flow of a second order fluid," Phys. Fluids 11, 5-9 (1968).

${ }^{25}$ K. Sadeghy, S. M. Taghavi, N. Khabazi, M. Mirzadeh, and I. Karimfazli, “On the use of hydrodynamic instability test as an efficient tool for evaluating viscoelastic fluid models," Adv. Stud. Theor. Phys. 1, 367-379 (2007).

${ }^{26}$ R. Sureshkumar and A. N. Beris, "Linear stability analysis of viscoelastic Poiseuille flow using an Arnoldi-based orthogonalization algorithm," J. NonNewtonian Fluid Mech. 56, 151-182 (1995).

${ }^{27}$ M. Zhang, I. Lashgari, T. A. Zaki, and L. Brandt, "Linear stability analysis of channel flow of viscoelastic Oldroyd-B and FENE-P fluids," J. Fluid Mech. 737, 249-279 (2013).

${ }^{28}$ G. K. Batchelor, An Introduction to Fluid Dynamics (Cambridge Mathematical Library Cambridge University Press, 2000).

${ }^{29} \mathrm{~W}$. Tollmien, "Über die entstehung der turbulenz (the production of turbulence)," in Vorträge aus dem Gebiete der Aerodynamik und verwandter Gebiete, edited by A. Gilles, L. Hopf, and T. V. Kármán (Springer, Berlin, Heidelberg, 1930), pp. 18-21.

${ }^{30}$ P. J. Schmid and D. S. Henningson, Stability and Transition in Shear Flows, Applied Mathematical Sciences (Springer, New York, 2000).

${ }^{31} \mathrm{M}$. Cracco, "Linear stability and transient behaviour of viscoelastic fluids in boundary layers," Ph.D. thesis, Cardiff University, 2019.

${ }^{32}$ T. Ellingsen and E. Palm, "Stability of linear flow," Phys. Fluids 18, 487-488 (1975). 
${ }^{33}$ L. N. Trefethen, Spectral Methods in MATLAB (Society for Industrial and Applied Mathematics, 2000).

${ }^{34}$ W. O. Criminale, T. L. Jackson, and R. D. Joslin, in Theory and Computation of Hydrodynamic Stability, Cambridge Monographs on Mechanics (Cambridge University Press, 2003).

${ }^{35} \mathrm{~N}$. Hoda, M. R. Jovanović, and S. Kumar, "Energy amplification in channel flows of viscoelastic fluids," J. Fluid Mech. 601, 407-424 (2008).

${ }^{36}$ B. K. Lieu, M. R. Jovanović, and S. Kumar, "Worst-case amplification of disturbances in inertialess Couette flow of viscoelastic fluids," J. Fluid Mech. 723, 232-263 (2013).
${ }^{37}$ J. Page and T. A. Zaki, "Streak evolution in viscoelastic Couette flow," J. Fluid Mech. 742, 520-551 (2014).

${ }^{38}$ N. Burshtein, K. Zografos, A. Q. Shen, R. J. Poole, and S. J. Haward, "Inertioelastic flow instability at a stagnation point," Phys. Rev. X 7, 041039 (2017).

${ }^{39}$ L. H. Gustavsson, "Initial-value problem for boundary layer flows," Phys. Fluids 22, 1602-1605 (1979).

${ }^{40} \mathrm{P}$. Corbett and A. Bottaro, "Optimal perturbations for boundary layers subject to stream-wise pressure gradient," Phys. Fluids 12, 120-130 (2000).

${ }^{41}$ P. J. Schmid, "Linear stability theory and bypass transition in shear flows," Phys. Plasmas 7, 1788-1794 (2000). 